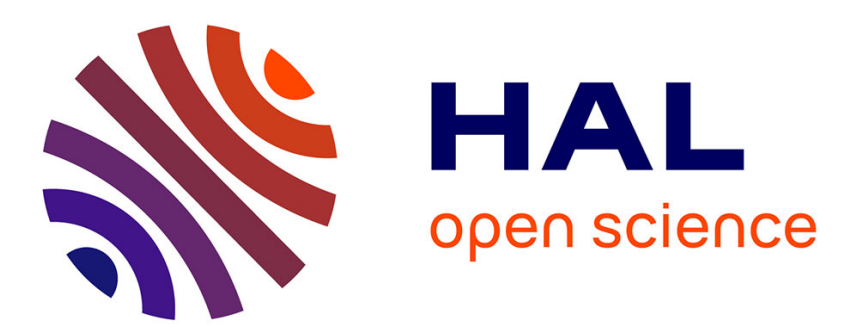

\title{
From anisotropy of dielectric tensors to birefringence: a quantum mechanics approach
}

\author{
Michel Rérat, Philippe D'arco, Valentina Lacivita, Fabien Pascale, Roberto
}

Dovesi

\section{- To cite this version:}

Michel Rérat, Philippe D'arco, Valentina Lacivita, Fabien Pascale, Roberto Dovesi. From anisotropy of dielectric tensors to birefringence: a quantum mechanics approach. Rendiconti Lincei. Scienze Fisiche e Naturali, 2020, 31 (3), pp.835-851. 10.1007/s12210-020-00931-9 . hal-02907958

\section{HAL Id: hal-02907958 \\ https://hal.science/hal-02907958}

Submitted on 2 Oct 2020

HAL is a multi-disciplinary open access archive for the deposit and dissemination of scientific research documents, whether they are published or not. The documents may come from teaching and research institutions in France or abroad, or from public or private research centers.
L'archive ouverte pluridisciplinaire HAL, est destinée au dépôt et à la diffusion de documents scientifiques de niveau recherche, publiés ou non, émanant des établissements d'enseignement et de recherche français ou étrangers, des laboratoires publics ou privés. 
See discussions, stats, and author profiles for this publication at: https://www.researchgate.net/publication/343238063

\section{From anisotropy of dielectric tensors to birefringence: a quantum mechanics approach}

Article in Rendiconti Lincei. Scienze Fisiche e Naturali · July 2020

DOI: 10.1007/s12210-020-00931-9

CITATIONS

5 authors, including:

Michel Rérat

Université de Pau et des Pays de l'Adour

161 PUBLICATIONS 4,654 CITATIONS

SEE PROFILE

Valentina Lacivita

Università degli Studi di Torino

20 PUBLICATIONS 265 CITATIONS

SEE PROFILE

\section{READS}

74

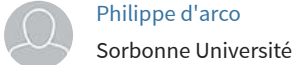

71 PUBLICATIONS 2,501 CITATIONS

SEE PROFILE

Fabien Pascale

3. University of Lorraine

41 PUBLICATIONS 2,224 CITATIONS

SEE PROFILE

Some of the authors of this publication are also working on these related projects:

$\mathrm{Ab}$ initio calculation of response properties of periodic systems and implementation of the frequency-dependent CPHF(KS) methods in the Crystal code (www.crystal.unito.it). View project

Statistics and benchmarking View project 


\title{
2 From anisotropy of dielectric tensors to birefringence: a quantum mechanics approach
}

4 Michel Rérat ${ }^{1}$ (1) $\cdot$ Philippe D'Arco ${ }^{2} \cdot$ Valentina Lacivita $^{3,4}$ (C) $\cdot$ Fabien Pascale $^{5} \cdot$ Roberto Dovesi $^{6}$

Received: 11 February 2020 / Accepted: 3 July 2020

(c) Accademia Nazionale dei Lincei 2020

\begin{abstract}
The way quantum mechanical ab initio computer codes allow to compute, through perturbation theory (the so-called SC-CP, self-consistent coupled-perturbed scheme), many properties resulting from the interaction of the electric field with a crystalline system is illustrated. The polarizability, which leads to the dielectric tensors as well as to the refractive indices and to he birefringence of materials, is the simplest on this list. Higher order tensors, like the first and second hyperpolarizabilities, can be obtained as well with the CRYSTAL code here used. These properties, resulting from the Taylor expansion of the total energy of the solid as a function of the electric field, belong to a large family of phenomena generated by combining in different ways the frequencies of the fields. Second-harmonic generation (SHG), Pockels effect, intensity-dependent refractive index (IDRI), and other quantities now accessible to experiment can be computed at a relatively low cost and with high accuracy.
\end{abstract}

Keywords Refractive index $\cdot$ Birefringence $\cdot$ (Non)linear electric susceptibility tensor · Anisotropy $\cdot$ Quantum mechanical simulation $\cdot$ CRYSTAL code $\cdot$ Gaussian-type basis set

This paper is the peer-reviewed version of a contribution presented at the Conference on Anisotropic Properties of Matter, organized by Giovanni Ferraris and held at Accademia Nazionale dei Lincei in Rome, October 16-17, 2019.

Michel Rérat

michel.rerat@univ-pau.fr

1 Université de Pau et des Pays de l'Adour, E2S UPPA, CNRS, IPREM, 2 av. président P. Angot, 64053 Pau, France

2 Sorbonne Université, CNRS-INSU, ISTeP UMR 7193, 75005 Paris, France

3 Advanced Materials Lab, Samsung Research America, 10 Wilson Rd., Cambridges, MA 02138, USA

4 Sorbonne Université, CNRS, IMJ UMR 7586, 75005 Paris, France

5 Université de Lorraine-Nancy, CNRS, Laboratoire de Physique et Chimie Théoriques, UMR 7019, Vandoeuvre-les-Nancy, France

6 Dipartimento di Chimica, Nanostructured Interfaces and Surfaces (NIS) Centre of Excellence, Università di Torino, Via P. Giuria 7, 10125 Turin, Italy

\section{Introduction}

In this contribution, we illustrate the way modern quantum mechanical methods allow to compute the (hyper)polarizability tensors and, consequently, optical properties such as the refractive index and birefringence, through which the anisotropy of the physical properties of crystalline compounds manifests itself.

The properties mentioned above can be obtained by investigating the interaction of the electromagnetic field with a periodic infinite system (the model implies, without serious consequences, that the crystalline compound is infinite).

The equations describing this interaction can be formulated at various levels (for example: relativistic or non-relativistic quantum mechanics). As none of these equations can be solved exactly, many approximations must be introduced, whose importance should be discussed carefully, and one should possibly verify numerically how severe these approximations are.

In a very broad sense, all these equations are solved by performing at various steps series expansions, so that the differential equations transform in matrix equations.

This essentially requires: (a) to compute matrix elements (these, in turn, are the sum of integrals, many of which are

\begin{tabular}{|l|l|l|l|l|}
\hline Journal : Large 12210 & Article No : 931 & Pages : 17 & MS Code : 931 & Dispatch : 20-7-2020 \\
\hline
\end{tabular}


bielectronic four center six dimensional integrals); (b) sum, multiply, and diagonalize matrices that can easily reach very large dimensions $\left(10^{3}-10^{6}\right.$, as typical cases).

The larger the matrices, the more accurate the calculation.

Obviously, this kind of linear algebra requires the use of computers (clusters of PC) or supercomputers containing $10^{2}-10^{4}$ processors.

The starting point for describing the crystalline system is the (stationary, or time-independent) Schrödinger's equation:

$\hat{\mathrm{H}} \Psi=\mathscr{E} \Psi$,

where $\hat{\mathrm{H}}$ is an operator, called Hamiltonian in memory of classical mechanics developed by Lagrange (1736-1813) and then Hamilton (1805-1865), and $\mathscr{E}$ the energy associated to the wavefunction $\Psi$.

The real limit of this equation is that $\hat{\mathrm{H}}$ and $\Psi$ depend on many variables as atomic positions, that is $\Psi \equiv \Psi\left(\mathbf{r}_{1}, \mathbf{r}_{2}, \mathbf{r}_{3}, \ldots, \mathbf{r}_{N}\right)$. In the case of an infinite crystalline system, $N$ goes to infinity. But also for a molecule like, say, benzene, containing 12 atoms and 42 electrons, for a total of 162 Cartesian coordinates (plus the spin), the Schrödinger's equation cannot be solved exactly, and its approximate solution requires a huge amount of skills and computational effort. We will not dwell on the techniques, hypotheses, and approximations that bring this intractable problem to something that can be tackled. The interested reader can refer to several excellent textbooks covering quantum mechanics and computational chemistry methods. We simply mention that these approximations bring to the so-called Hartree-Fock or Kohn-Sham methods, in which a single particle (say electron) is moving in the field created by all the other electrons (whose wavefunction is unknown; mean field theory). This implies, in turn, that these equations must be solved through a self-consistent field (SCF) scheme.

We can now suppose that we are able to describe with reasonable accuracy the ground state of a crystalline system.

We are then faced with the problem of the description of the electromagnetic field, and of its interaction with the solid. This interaction is described through a well-known tool of quantum mechanics, namely the perturbation theory, that takes the form of a Taylor expansion of the system's energy in powers of the electric field. As usual, the series is truncated after a few terms due to (a) computational costs and (b) hopefully, the rapid convergence. Also in this case, the solution of the resulting equations requires an iterative scheme. At the very end, the crucial points (crucial due to numerical accuracy and computational cost) are: evaluation of multicenter integrals and multiplication of very large matrices.

We are then considering a crystalline solid, and an electric field operating on it. What is the information that can be obtained as a response of the system to this perturbation? Let us consider the Taylor expansion of the total bulk energy of the system with respect to the field amplitude $\mathbf{E}_{0}$, truncated to the fourth order:

$$
\begin{aligned}
\mathscr{E}= & \mathscr{E}_{0}-\boldsymbol{\mu}_{0} \cdot \mathbf{E}_{0}-\frac{1}{2 !} \boldsymbol{\alpha}_{0} \mathbf{E}_{0} \otimes \mathbf{E}_{0}-\frac{1}{3 !} \boldsymbol{\beta}_{0} \mathbf{E}_{0} \otimes \mathbf{E}_{0} \otimes \mathbf{E}_{0} \\
& -\frac{1}{4 !} \gamma_{0} \mathbf{E}_{0} \otimes \mathbf{E}_{0} \otimes \mathbf{E}_{0} \otimes \mathbf{E}_{0},
\end{aligned}
$$

where $\boldsymbol{\mu}_{0}, \boldsymbol{\alpha}_{0}, \boldsymbol{\beta}_{0}$, and $\boldsymbol{\gamma}_{0}$ are the permanent dipole moment, polarizability, and first and second hyperpolarizabilities of the free system, respectively (the conventional negative sign is such that the dipole moment is defined as the sum over the charges multiplied by their position, and such that the polarizability of the ground state is positive). The symbol $\otimes$ indicates the outer product of vectors. Given the electric field conversion coefficient from the atomic units (a.u.) system to the international system of units (SI): 1 a.u. $=5.14 \times 10^{11} \mathrm{Vm}^{-1}$, a large static field of say $50 \mathrm{kVcm}^{-1}$ (maximum field amplitude that can be applied with electrodes at the surface of a slab before electric breakdown), is smaller than $10^{-5}$ a.u.. If we apply a field of this amplitude to a molecule, say water, with a polarizability equal to $\sim 10 \mathrm{bohr}^{3}$ (the polarizability unit is equivalent to a volume in a.u.), the energy variation due to the polarization would be equal to $10^{-9} \mathrm{E}_{h}$. As the ratio between the terms appearing in Eq. $2\left(\boldsymbol{\beta}_{0} / \boldsymbol{\alpha}_{0}, \boldsymbol{\gamma}_{0} / \boldsymbol{\beta}_{0}\right)$ is generally smaller than $10^{3}$ a.u., to be multiplied by an additional field intensity of $10^{-5}$, it is clear that contributions approach rapidly the numerical accuracy limit of quantum mechanical calculations. This is why, $\boldsymbol{\beta}_{0}$ and $\boldsymbol{\gamma}_{0}$ coefficients have been considered in the past of low interest, and terms as $\boldsymbol{\delta}_{0}$, corresponding to power five of $\mathbf{E}_{0}$, have been neglected in Eq. 2 . However, if the expanded quantity is not the total energy, but some higher term evaluated analytically, then the power of $\mathbf{E}_{0}$ for obtaining $\boldsymbol{\beta}_{0}$ and $\boldsymbol{\gamma}_{0}$ is lower. This is the case, for example, when the polarizability $\boldsymbol{\alpha}_{0}$ is computed analytically, with a reduction by 2 of the power of $\mathbf{E}_{0}$. Moreover, lasers with much higher intensity than static electric fields are now available, which can allow to access experimentally many non-negligible second- and third-order non-linear optical (NLO) effects.

It should be stressed that Eq. 2 can provide a lot of information:

(a) as $\mathbf{E}_{0}$ is a vector with three components, $\mathrm{E}_{x}, \mathrm{E}_{y}$, and $\mathrm{E}_{z}$, it turns out that $\boldsymbol{\mu}_{0}, \boldsymbol{\alpha}_{0}, \boldsymbol{\beta}_{0}$, and $\boldsymbol{\gamma}_{0}$ are tensors of rank 1 , 2,3 , and 4 , respectively, whose components can vary from case to case, allowing access to important specific features.

(b) The electric fields appearing in Eq. 2 can be different from each other (say $\mathbf{E}_{1}, \mathbf{E}_{2} \ldots$ ), and combined in different ways. 
(c) Obviously, the various electric fields can depend on frequency as in light radiation, $\mathbf{E} \equiv \mathbf{E}(\omega)=\mathbf{E}_{0} \cos (\omega t)$ with the angular $\omega$-frequency of the corresponding photon ( $\hbar \omega$ with $\hbar=h / 2 \pi, h$ being the Planck constant equal to $6.63 \times 10^{-34} \mathrm{Js}$ ), further increasing the number of possible ways of perturbing the system. For example, in the second-harmonic generation (SHG) experiments, interaction of light with matter can provide one scattered photon of energy $\hbar 2 \omega$ from two photons of energy $\hbar \omega$, the intensity of the scattering light depending on the frequency-dependent first hyperpolarizability, $\boldsymbol{\beta}(-2 \omega ; \omega, \omega)$, of the material, as we will see later on.

(d) As a corollary to point (c), the frequency can be used for perturbing both nuclei and electrons, or just the latter.

The following section will deepen some of the main topics introduced here.

\section{Methods}

\subsection{Dipole moment and (hyper)polarizability}

\subsubsection{Definitions}

The dipole moment $\boldsymbol{\mu}$ of a finite system is a vector defined, in the atomic unit system, as:

$\boldsymbol{\mu}=\sum_{N} Z_{N} \mathbf{r}_{N}-\int \mathbf{r} \rho(\mathbf{r}) \mathrm{d} \mathbf{r}$,

where the absolute value of the electron charge is equal to 1 ( $e=-1$ a.u.). $Z_{N}$ and $\mathbf{r}_{\mathbf{N}}$ are the nuclear charge and position of the $N$ th atom, and $\rho(\mathbf{r})$ is the electron charge density in $\mathbf{r}$. For a given geometry, $Z_{N}$ and $\mathbf{r}_{N}$ are fixed, and the value of the dipole moment can be obtained if the electron density is known in the whole space. In quantum chemistry, this latter, which is the square of a wave function, $\Psi$, describing the electronic state of the system (generally its ground state) can be obtained by solving the time-independent Schrödinger's equation (see Eq. 1). Using Dirac's notation, $\int \mathbf{r} \rho(\mathbf{r}) d \mathbf{r}$ in Eq. 3 can now be replaced by $\langle 0|\mathbf{r}| 0\rangle$ where $\langle 0|$ and $|0\rangle$ represent the bra and ket of the ground state.

In the presence of a time-dependent electromagnetic $(\mathbf{E}(\mathbf{r}, \mathrm{t}), \mathbf{B}(\mathbf{r}, \mathrm{t}))$ field, the expression of $\hat{\mathrm{H}}$ becomes:

$\hat{\mathrm{H}}=\frac{1}{2 m}\left(\mathbf{p}-\frac{e}{c} \mathbf{A}(\mathbf{r}, t)\right)^{2}+e U(\mathbf{r}, t)+V_{C}(\mathbf{r})$,

where $\mathbf{p}$ and $\mathbf{r}$ are the momentum and position of the electron, $m$ its mass ( $m=1$ a.u.), $c$ the speed of light ( $c=137$. a.u.), and $V_{C}$ the Coulomb potential. The electromagnetic field is defined from the vector $\mathbf{A}(\mathbf{r}, \mathrm{t})$ and scalar $\mathrm{U}(\mathbf{r}, \mathrm{t})$ potentials via Maxwell's equations:

$\mathbf{E}(\mathbf{r}, t)=-\frac{\partial \mathbf{A}(\mathbf{r}, t)}{\partial t}-\nabla U(\mathbf{r}, t)$

$\mathbf{B}(\mathbf{r}, t)=\boldsymbol{\nabla} \times \mathbf{A}(\mathbf{r}, t)$,

and there is an infinite number of $(\mathbf{A}(\mathbf{r}, \mathrm{t}), \mathrm{U}(\mathbf{r}, \mathrm{t}))$ couples describing one electromagnetic field. Obviously, the solutions of the time-dependent Schrödinger's equation must be independent from the choice of the so-called gauge. This is indeed the case of the energy $E$-eigenvalue as well as of $|\Psi(\mathbf{r}, t)|^{2}$. Only the phase of the wave function depends on the choice of the gauge.

For a static electric field: $\mathbf{E}(\mathbf{r})=\mathbf{E}_{0} e^{l \mathbf{q} \cdot \mathbf{r}}$ with a small wave number: $q=2 \pi / \lambda$ compared to $\mathbf{r}$ (i.e., for $\lambda$-wavelength much larger than the size of the studied system: $\lambda \gg 1 \AA$ ), $\mathbf{E}(\mathbf{r})$ is generally defined from the scalar potential only as follows:

$\mathbf{E}(\mathbf{r})=-\nabla U(\mathbf{r})$,

which leads for a constant field $\mathbf{E}_{0}$ to:

$\hat{\mathrm{H}}=\frac{\mathbf{p}^{2}}{2 m}+V_{C}(\mathbf{r})-e \mathbf{r} \cdot \mathbf{E}_{0}=\hat{\mathrm{H}}_{0}-e \mathbf{r} \cdot \mathbf{E}_{0}$

in the electric dipole moment approximation for finite systems ( $0 \mathrm{D}$ or molecules), $\hat{\mathrm{H}}_{0}$ being the Hamiltonian operator of the unperturbed system.

The energy $\mathscr{E}$ of the molecule in presence of the $\mathbf{E}_{0}$-field can be developed as a Taylor series as shown in Eq. 2; similarly, for the dipole moment, we have:

$$
\begin{aligned}
\boldsymbol{\mu}= & -\frac{\mathrm{d} \mathscr{E}}{\mathrm{d} \mathbf{E}_{0}}=\mu_{0}+\boldsymbol{\alpha}_{0} \mathbf{E}_{0}+\frac{1}{2 !} \boldsymbol{\beta}_{0} \mathbf{E}_{0} \otimes \mathbf{E}_{0} \\
& +\frac{1}{3 !} \gamma_{0} \mathbf{E}_{0} \otimes \mathbf{E}_{0} \otimes \mathbf{E}_{0}+\cdots,
\end{aligned}
$$

where $\boldsymbol{\mu}_{0}$ is the permanent dipole moment of the molecule, the linear response $\boldsymbol{\alpha}_{0}$ is called the polarizability, and $\boldsymbol{\beta}_{0}$ and $\gamma_{0}$ are the first and second hyperpolarizabilities. The previous electric responses to the electric field are intrinsic properties of the molecule: they are equal to the first, second, and third derivatives of the induced dipole moment $\left(\boldsymbol{\mu}-\boldsymbol{\mu}_{0}\right)$ with respect to the field $\mathbf{E}_{0}$, at zero field $\left(\mathbf{E}_{0} \rightarrow \mathbf{0}\right)$. Moreover, being $\boldsymbol{\mu}_{0}$ and $\mathbf{E}_{0}$ vectors, $\boldsymbol{\alpha}_{0}, \boldsymbol{\beta}_{0}$ and $\boldsymbol{\gamma}_{0}$ are tensors of rank 2,3 , and 4 , respectively.

In the case of a frequency-dependent electric field $\left(\mathbf{E}(\omega)=\mathbf{E}_{0} \cos \omega t\right)$, the linear term of the dipole moment induced by the oscillating field is oscillating at the same frequency of the field (forced oscillation if the field frequency is small compared to proper resonance frequencies of the system) leading to a frequency-dependent polarizability, $\boldsymbol{\alpha}(\omega)$. For a molecule in its electronic ground state $(|0\rangle)$,

\begin{tabular}{|l|l|l|l|l|}
\hline Journal : Large 12210 & Article No : 931 & Pages : 17 & MS Code : 931 & Dispatch : 20-7-2020 \\
\hline
\end{tabular}


the "dynamic" polarizability is a sum of two contributions that can be separately calculated in the Born-Oppenheimer approximation:

1. The electronic $\left(\boldsymbol{\alpha}^{e}\right)$ contribution, for a fixed $\left(\mathbf{r}_{N}\right)$ geometry:

$$
\boldsymbol{\alpha}^{e}(\omega)=\sum_{n \neq 0}^{\infty} 2 \omega_{n} \frac{\boldsymbol{\mu}_{n} \otimes \boldsymbol{\mu}_{n}}{\omega_{n}^{2}-\omega^{2}},
$$

where $\omega_{n}=\mathscr{E}_{n}-\mathscr{E}_{0}$ are the allowed transition energies from the $|0\rangle$-ground to $|n\rangle$-excited electronic states with $\mathscr{E}_{0}$ and $\mathscr{E}_{n}$ as corresponding eigenvalues of the unperturbed Hamiltonian operator $\hat{\mathrm{H}}_{0}$ (if we are interested in the polarizability of the ground state) and $\boldsymbol{\mu}_{n}=\langle 0|\mathbf{r}| n\rangle$ is the corresponding transition dipole moment.

The associated mean value oscillator strengths:

$$
f_{n}=\frac{2}{3} \omega_{n}\langle 0|\mathbf{r}| n\rangle^{2}
$$

are such that $\sum_{n} f_{n}$ is equal to the number of electrons involved in these transitions, and the $\left(\omega_{n}, f_{n}\right)$ couples reproduce the UV-visible spectrum.

See the work of Orr and Ward (1971) for the expression of hyperpolarizabilities.

2. The ionic or nuclear relaxation $\left(\boldsymbol{\alpha}^{n r}\right)$ contribution:

$$
\boldsymbol{\alpha}^{n r}(\omega)=\sum_{i=1}^{3 N-6} \frac{\sigma_{i} \otimes \sigma_{i}}{\omega_{i}^{2}-\omega^{2}},
$$

where $\omega_{i}$ is the frequency of the normal mode $\left(Q_{i}\right)$ and $\boldsymbol{\sigma}_{i}$ the Born charge $\left(\mathrm{d} \mu_{0} / \mathrm{d} Q_{i}\right.$ with $\boldsymbol{\mu}_{0}$ as defined in Eq. 3 at zero field) obtained at the equilibrium geometry. The $\left(\omega_{i}, \sigma_{i}\right)$ couples reproduce the infrared (IR) spectrum.

We refer to the work of Kirtman and Luis (2010) for the treatment of the vibrational hyperpolarizabilities that can be evaluated from the IR and (hyper) Raman spectra.

$\boldsymbol{\alpha}^{n r}$ is zero in purely covalent materials as diamond or silicon, and also negligible for other ionic materials if the field frequency $(\omega)$ corresponds to the UV-visible range of energy $\omega \gg \omega_{i}$. Indeed, the Born charge $\mathrm{d} \boldsymbol{\mu}_{0} / \mathrm{d} Q_{i}$, which is actually a charge divided by the square root of ion mass, is small with respect to 1 a.u., and $\sigma_{i}^{2} /\left(\omega_{i}^{2}-\omega^{2}\right) \simeq-\sigma_{i}^{2} / \omega^{2}$, which is then negative, becomes very small in absolute values compared to the static vibrational polarizability contribution: $\sigma_{i}^{2} / \omega_{i}^{2}$.

Also the vibrational contribution to $\boldsymbol{\beta}$ and $\boldsymbol{\gamma}$ can be neglected in the UV-visible frequency range for the same reason as for $\boldsymbol{\alpha}$, if all the external fields are frequencydependent. The exception is the particular case of the intensity-dependent refractive index, IDRI, a process depending on $\gamma(-\omega ; \omega,-\omega, \omega)$, that includes terms with opposite sign phases, $\pm \omega$, generating then a static field and a vibrational contribution.

\subsubsection{Calculation of the microscopic response properties}

The electronic contribution to the polarizability (Eq. 10) is a second-order perturbation energy, the expression of which is a sum extended to an infinite number of excited state terms, a series which generally converges very slowly. Moreover, it also depends on the continuum. For example, if we use all the true (and well known) discrete spectral states of the $\mathrm{H}$ atom, its polarizability value is less than $4 \mathrm{bohr}^{3}$, while its exact value is $9 / 2$ bohr $^{3}$ (without taking into account the relativistic effect) (Coulson 1941; McDowell 1976; Traini 1996; Bishop 1999).

Fortunately, it is not necessary to know all the excited discrete and continuum states of the electronic system to obtain a "good" static polarizability value, or dynamic polarizabilities for a field frequency smaller than the first resonance one. The coupled-perturbed Hartree-Fock (CPHF) method proposed by Hurst et al. (1988), consisting in the independent-particle model using relaxed occupied and virtual orbitals via a self-consistent process in the presence of the external field, generally leads to results in good agreement with the experiments, in particular when a density functional theory (DFT) Hamiltonian with a percentage of the exact or Hartree-Fock (HF) non-local exchange potential is used, as is the case of the hybrid B3LYP (Becke 1993; Lee et al. 1988) functional (20\% of HF exchange). Indeed, response properties which are n-order perturbation energies depend strongly on the band gap (which determines the lowest transition energy values, $\omega_{n} \geq$ gap, on the denominator of Eq. 10 for electronic polarizability, and of hyperpolarizabilities), generally too large with HF (Evarestov 2007) and too small with pure DFT (Yakovkin and Dowben 2007), but reasonably described by hybrids (Garza and Scuseria 2016).

\subsubsection{Infinite periodic systems}

Expression 8 of the Hamiltonian, that includes the position operator, $\mathbf{r}$, as the perturbation operator associated to the external field, comes from the dipole moment approximation that can be used for finite systems (molecules) in the presence of a constant field, or a field with a large $\lambda$ wavelength with respect to the size of the electronic system $(\lambda \gg 1 \AA)$.

For infinite periodic systems, the electric potential $\mathbf{r} \cdot \mathbf{E}_{0}$ becomes infinite at $( \pm \infty)$, where the electronic density $\rho$ is finite, so that the wavefunction is not square summable in Eq. 3. Moreover, infinite periodic systems described by symmetry translated cells and for which the crystalline orbitals (CO) are combinations of Bloch functions following Born von Karman (BVK) conditions (last cell in each direction is bound with the first one) have an ill-defined dipole moment. 
Indeed, what is the dipole moment of a polymer $(\mathrm{AB})_{n \rightarrow \infty}$, that of $\mathrm{AB}:-(\mathrm{A}-\mathrm{B})-(\mathrm{A}-\mathrm{B})-(\mathrm{A}-\mathrm{B})-$ or the one of $\mathrm{BA}$ : $-\mathrm{A})-(\mathrm{B}-\mathrm{A})-(\mathrm{B}-\mathrm{A})-(\mathrm{B}-$ ?

Actually $\mathbf{r}$, which is a non-periodic potential, is not Hermitian in the BVK CO basis set depending of the $\mathbf{k}$ points of the reciprocal space. Then, we must restart from the expression of an external electric field described by a scalar potential gauge: $\mathbf{E}=\mathbf{E}_{0} e^{\imath \mathbf{q} \cdot \mathbf{r}}$, where $\mathbf{E}_{0}$ is the field amplitude and $q$ its wave number $2 \pi / \lambda$, and determine dipole moment transitions between $i(\mathbf{k})$ and $j\left(\mathbf{k}^{\prime}\right)$ COs involved in the field perturbation at the $\mathbf{q} \rightarrow \mathbf{0}$ limit for a constant field. Using the momentum conservation: $\mathbf{q}=\mathbf{k}^{\prime}-\mathbf{k}$, it follows that the dipole moment operator becomes (Blount et al. 1962; Otto 1992; Rérat et al. 2008):

$\hat{\Omega}_{k}=\mathbf{r}+\imath \nabla_{k}=-\imath e^{-\imath \mathbf{k} \cdot \mathbf{r}} \nabla_{k} e^{\imath \mathbf{k} \cdot \mathbf{r}}$,

and the perturbative Hamiltonian (multiplied by the constant $\mathbf{E}_{0}$ field) is Hermitian and remains block diagonal in the reciprocal space for $\lambda \gg 1 \AA$.

The basic equations of the self-consistent coupled-perturbed (SC-CP) computational scheme for periodic systems can be found in Ferrero et al. (2008a, b) for the CPHF calculation of (hyper)polarizabilities, adapted to Kohn-Sham Hamiltonian (CPKS) in Orlando et al. (2010) and to frequencydependent electric fields in Ferrari et al. (2015), Rérat et al. (2015), Maschio et al. (2015), whereas many numerical examples are reported in Sect. 3.

\subsection{Refractive index}

In this section, we are going to look at the refractive index of materials, which is a macroscopic property depending on the (hyper)polarizability described in the previous section, as we will see further. Let us look first at the definition of this optical property and then at the resulting birefringence of materials.

\subsubsection{Definition}

Electric induction (D) and magnetic excitation $(\mathbf{H})$ fields are related to the frequency $(\omega)$-dependent electromagnetic $(\mathbf{E}, \mathbf{B})$ field as follows (Condon 1937):

$\mathbf{D}=\epsilon_{r} \epsilon_{0} \mathbf{E}-\frac{\xi}{c} \frac{\partial \mathbf{H}}{\partial t}$

$\mathbf{B}=\boldsymbol{\mu}_{r} \mu_{0} \mathbf{H}+\frac{\boldsymbol{\xi}}{c} \frac{\partial \mathbf{E}}{\partial t}$

where $\boldsymbol{\epsilon}_{r}$ and $\boldsymbol{\mu}_{r}$ are the relative electric permittivity and magnetic permeability (matrices) of the medium, respectively; $\epsilon_{0}$ and $\mu_{0}$ are the permittivity and permeability (constants) of the vacuum, such that $\mu_{0} \epsilon_{0} c^{2}=1$; and $\xi$ is the chirality (matrix) responsible of the rotation angle of a polarized electromagnetic field.

Then, for materials with $\boldsymbol{\mu}_{r} \simeq \mathbf{1}$ (low magnetic permeability) and $\boldsymbol{\xi}=\mathbf{0}$ (no chirality), the refractive index is (Condon 1937):

$\mathbf{n}=\left(\left(\boldsymbol{\mu}_{r} \boldsymbol{\epsilon}_{r}\right)^{-\frac{1}{2}} \pm \omega \xi\right)^{-1} \simeq \sqrt{\boldsymbol{\epsilon}_{r}}$

\subsubsection{Optical indicatrix and birefringence}

As seen above, the relative dielectric tensor $\epsilon_{r}$ is a secondrank symmetric tensor usually represented by a Hermitian matrix. This matches an ellipsoid (Nye 1985), the optical indicatrix, whose equation is:

$\frac{X^{2}}{n_{X}^{2}}+\frac{Y^{2}}{n_{Y}^{2}}+\frac{Z^{2}}{n_{Z}^{2}}=1$

and with semi-axis lengths given by the square roots of the dielectric tensor eigenvalues, $n_{i}=\sqrt{\epsilon_{i}}(i=X, Y, Z)$, corresponding to the principal refractive indices of the medium. We will assume that the indices along the semi-axis $X, Y, Z$, are ordered by increasing value.

Hence, the phenomenon of birefringence is estimated as the difference $\delta=n_{Z}-n_{X}$. In principle, all crystals are birefringent and the specific indicatrix properties depend on the crystal symmetry. However, some special directions-the optical axes-exist which select as many circular sections within the indicatrix. For cubic minerals, which are optically isotropic, the optical indicatrix (see Fig. 1) is a sphere (null birefringence) defined by a unique refractive index $n$. The uniaxial optical indicatrix of tetragonal, hexagonal, or trigonal minerals is an ellipsoid of revolution characterized by two independent semi-axis of length $n_{\omega}$ and $n_{\epsilon}$. The axis of revolution of the indicatrix parallels the $c$-direction of the mineral and corresponds to $n_{\epsilon}$. Such ellipsoid possesses a single circular section perpendicular to the $c$-direction which corresponds to the optical axis. The birefringence is $\delta=\left|n_{\epsilon}-n_{\omega}\right|$. If $n_{\epsilon}>n_{\omega}\left(n_{\epsilon}=n_{Z}, n_{\omega}=n_{X}=n_{Y}\right)$ the indicatrix and the corresponding mineral are said uniaxial positive. If $n_{\epsilon}<n_{\omega}$ $\left(n_{\epsilon}=n_{X}, n_{\omega}=n_{Y}=n_{Z}\right)$, the indicatrix and the corresponding mineral are said uniaxial negative. Any other mineral is biaxial, having an optical ellipsoid with two circular sections (and as many optical axes) of indices $n_{Y}$ (see Fig. 1). $n_{X}$ and $n_{Z}$ lie on the plane of the optical axes and bisect the angles between them. Depending on whether the acute angle between the optical axes $(2 V)$ is bisected by $n_{Z}$ $\left(2 V=2 V_{Z}\right)$ or by $n_{X}\left(2 V=2 V_{X}\right)$, the crystal is said to be positive or negative. $2 V_{Z}+2 V_{X}=\pi$. The following equation defines the relationship between $\mathrm{V}$ and $n_{X}, n_{Y}$ and $n_{Z}$ :
380

381

382

383

384 


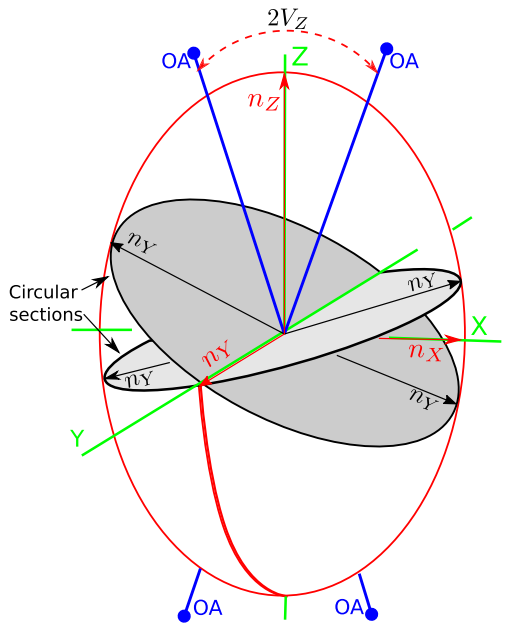

Fig. 1 Representation of the indicatrix of a positive biaxial crystals. The directions of the principal axes of the ellipsoid $X, Y$, and $Z$ are represented by a thick green line, and the associated red thin arrows indicate the corresponding refractive indices $n_{X}, n_{Y}$, and $n_{Z}$. The grey surfaces are the circular sections of the indicatrix, and their radius is constant and equals $n_{Y}$. The normals to the circular sections or optical axis (OA) form the acute $2 V_{Z}$ angle bisected by the $Z$ semi-axis. For uniaxial crystals $n_{X}=n_{Y}$, usually indicated as $n_{\omega}$, and $n_{Z}$ is indicated as $n_{\epsilon}$; the two circular sections merge in a single one orthogonal to $Z$

$\cos V=\frac{n_{X}}{n_{Y}} \sqrt{\frac{n_{Z}^{2}-n_{Y}^{2}}{n_{Z}^{2}-n_{X}^{2}}}$.

\subsubsection{Non-linear optics and birefringence}

Intense electric fields can also provide birefringence as demonstrated in the following.

Equation 14 for $\boldsymbol{\xi}=\mathbf{0}$ can also be written as follows:

$\mathbf{D}=\epsilon_{r} \epsilon_{0} \mathbf{E}=\epsilon_{0} \mathbf{E}+\mathbf{P}$,

where the polarization vector, $\mathbf{P}$, is the dipole moment per unit volume induced by the macroscopic field, and can be developed in a Taylor series as follows:

$\mathbf{P}=\epsilon_{0}\left(\chi^{(1)} \mathbf{E}+\chi^{(2)} \mathbf{E} \otimes \mathbf{E}+\chi^{(3)} \mathbf{E} \otimes \mathbf{E} \otimes \mathbf{E}+\cdots\right)$

where $\chi^{(n)}$ are the (non)linear electric susceptibility tensors of rank $(n+1)$, the units of which are the inverse of field to the $(n-1)$ th power.

Let us consider the field $\mathbf{E}$ produced by the light wave of frequency $\omega$ together with an external static electric field $\mathbf{E}_{0}$ :

$\mathbf{E}=\mathbf{E}_{0}+\mathbf{E}_{\omega} \cos \omega t$

where $\mathbf{E}_{\omega}$ is the vector amplitude of the wave. Then, a nonzero component value of $\chi^{(2)}$ leads to an additive term proportional to $\mathrm{E}_{0}$-modulus in the expression of the polarization vector which oscillates with the same $\omega$ frequency as the electromagnetic field:

$\mathbf{P}(\omega)=\varepsilon_{0}\left(\chi_{(-\omega ; \omega)}^{(1)}+2 \chi_{(-\omega ; \omega, 0)}^{(2)} \mathbf{E}_{0}\right) \mathbf{E}_{\omega} \cos \omega t$,

and induces a linear variation of the refractive index with respect to the modulus of $\mathbf{E}_{0}$ :

$$
\begin{aligned}
\mathbf{n}(\omega) & =\sqrt{\mathbf{1}+\chi_{(-\omega ; \omega)}^{(1)}+2 \chi_{(-\omega ; \omega, 0)}^{(2)} \mathbf{E}_{0}} \\
& \simeq \mathbf{n}_{0}(\omega)+\mathbf{n}_{1}(\omega) \mathbf{E}_{0}
\end{aligned}
$$

with

$\mathbf{n}_{0}(\omega)=\sqrt{\mathbf{1}+\chi_{(-\omega ; \omega)}^{(1)}}$ and $\mathbf{n}_{1}(\omega)=\chi_{(-\omega ; \omega, 0)}^{(2)} / \mathbf{n}_{0}(\omega)$.

This Pockels effect responsible of the birefringence: $\Delta \mathbf{n}=\mathbf{n}_{1} \mathbf{E}_{0}$, depends on the odd rank tensor, $\chi^{(2)}$, which is null for materials with an inversion symmetry. However, the birefringence of centrosymmetric materials can be seen if they own a large third-order non-linear $\chi^{(3)}$ susceptibility (tensor of rank four) and for intense electric fields. Indeed, it comes from the quadratic term in $\mathbf{E}$ in the polarization vector expression (Eq. 18). In the intensity-dependent refractive index (IDRI) Kerr effect (see Boyd 2003), an intense beam of light in the crystal can itself provide the modulating electric field:

$\mathbf{E}=\mathbf{E}_{\omega} \cos \omega t=\frac{1}{2} \mathbf{E}_{\omega}\left(\mathrm{e}^{\imath \omega t}+\mathrm{e}^{-\imath \omega t}\right)$

without the need for an external field to be applied. The product of frequency-dependent fields leads again to a polarization vector oscillating with the same frequency as the laser field:

$\mathbf{P}(\omega)=\varepsilon_{0}\left(\chi_{(-\omega ; \omega)}^{(1)}+\frac{3}{4} \chi_{(-\omega ; \omega,-\omega, \omega)}^{(3)} \mathbf{E}_{\omega}^{2}\right) \mathbf{E}_{\omega} \cos \omega t$,

and then, we have:

$$
\begin{aligned}
\mathbf{n}(\omega) & \simeq \mathbf{n}_{0}(\omega)+\frac{3}{8 n_{0}(\omega)} \chi_{(-\omega ; \omega,-\omega, \omega)}^{(3)} \mathbf{E}_{\omega}^{2} \\
& \simeq \mathbf{n}_{0}(\omega)+\mathbf{n}_{2}(\omega) \mathbf{I}
\end{aligned}
$$

with $\mathbf{I}$ the light intensity. In that case, the birefringence is given by $\mathbf{n}_{2}$, i.e., by the IDRI-Kerr non-linear electric $\chi_{(-\omega ; \omega,-\omega, \omega)}^{(3)}$ susceptibility.

A recent application referring to yttria-stabilized zirconia can be found in Marcaud et al. (2020). Several other NLO processes also appear when combining the Taylor development of the polarization vector shown in Eq. 18 with the field expression given in Eq. 19 (see Saleh and Teich 1991; Boulon 2001), as SHG linked to $\chi^{(2)}(-2 \omega ; \omega, \omega)$ and third harmonic generation (THG) linked to $\chi^{(3)}(-3 \omega ; \omega, \omega, \omega)$, but they do not affect the refractive index at $\omega$-frequency.

\begin{tabular}{|l|l|l|l|l|}
\hline Journal : Large 12210 & Article No : 931 & Pages : 17 & MS Code : 931 & Dispatch : 20-7-2020 \\
\hline
\end{tabular}




\subsubsection{Relation between (non)linear susceptibilities and (hyper)polarizabilities}

The macroscopic (non)linear optical properties, $\chi^{(n)}$, in the expression (Eq. 18) of the polarization vector are linked to the microscopic $\boldsymbol{\alpha}, \boldsymbol{\beta}, \boldsymbol{\gamma} \ldots$ (hyper)polarizability properties of a unit cell of the material, recalling that $\mathbf{P}$ is the dipole moment per unit cell volume $V$ induced by the mean (macroscopic) field (E) felt by the cell, and that can be also developed as follows:

$\mathbf{P}=\frac{1}{V}\left(\alpha \mathbf{E}+\frac{1}{2 !} \beta \mathbf{E} \otimes \mathbf{E}+\frac{1}{3 !} \gamma \mathbf{E} \otimes \mathbf{E} \otimes \mathbf{E}+\cdots\right)$.

The relative electric permittivity (or dielectric matrix) $\boldsymbol{\epsilon}_{r}$ at zero field is then linked to the polarizability $\boldsymbol{\alpha}$ of the unit cell:

$\varepsilon_{r}=\mathbf{1}+\chi^{(1)}=\mathbf{1}+\frac{1}{\varepsilon_{0}} \alpha / V$,

where $V$ is the unit cell volume. Similarly, we have: $\chi^{(2)}=\frac{1}{2 !} \frac{1}{\epsilon_{0}} \boldsymbol{\beta} / V$ and $\chi^{(3)}=\frac{1}{3 !} \frac{1}{\epsilon_{0}} \gamma / V$, with $1 / \varepsilon_{0}=4 \pi$ in atomic units.

It follows that the $\boldsymbol{\alpha}, \boldsymbol{\beta}$, and $\boldsymbol{\gamma}$ tensors obtained from the $\mathrm{SC}-\mathrm{CP}$ calculation discussed in Sect. 2.1.3 for infinite periodic systems allow to determine the (non)linear susceptibilities, $\chi^{(n)}$, and the refractive index and birefringence.

\section{Applications and comparison with experimental data}

The examples should provide an idea of the quality of the results that can be obtained by simulation, and in particular with the CRYSTAL code (Dovesi et al. 2017, 2018, 2020).

Before illustrating these examples, it should be mentioned that (obviously) the results depend deeply on the computational parameters; the most important are listed below:

- The variational basis set: in the following examples, a localized Gaussian basis set in split valence or multiple zeta forms, usually including polarization functions, is used. This is in general sufficient to provide accurate results.

- The level of the theory: all calculations are performed at the DFT level, and the most reliable, in our opinion, are obtained with hybrid functionals, containing a fraction of the exact Hartree-Fock exchange. However, as the superiority of one functional with respect to the others is frequently a matter of discussion, in some cases we will compute the same property with various functionals.
- The intrinsic numerical accuracy of the implemented algorithms, that in the CRYSTAL code is very high, so that its influence on the final results can be considered negligible.

One additional point must be underlined, when comparing simulation and experiments: the experimental determinations to be compared with are, in many cases, scarce, or affected by large uncertainty, so that error bars on the two sides (simulation and experiment) should be considered. Just to mention an example: the complete determination of all components of a tensor (and many of the ones mentioned above are third or fourth-order tensors, with many components) requires that the experiment is repeated with different orientations of the crystal, possibly with various polarization of the light. The obtained results are often not directly the specific constant to be inserted in the tensor table, but a linear combination of them generating a system of (linear) equations, whose solution (in particular when small and large numbers are involved) can produce strongly correlated final values. The uncertainty is increased in some cases by the fact that, intrinsically, the experiment is unable to determine the signs of the constants.

One advantage of simulation is that all components of the tensors are determined in a single shot, so that the accuracy of the various terms is the same.

The drawback of simulation is that quantum mechanical calculations refer to $T=0 \mathrm{~K}$, so that the effect of temperature can just be guessed, or evaluated a posteriori with simple and, in general, not very accurate tools.

A few more words about anisotropy: each one of the properties listed above, when referred to a gas or a liquid, reduces to the trace (for order two tensors) or to a few invariants (for higher order tensors). In the solid state, on the contrary, all specificity related to orientation is contained in the tensor. Tensors are defined with reference to a cartesian frame (there are rules for defining the orientation of the lattice vectors with respect to the cartesian frame), so that the constants take the following form, for a fourth-order tensor: $T_{i j k l}$, where $i, j, k, l$ can be $x$, or $y$ or $z$. The first manifestation of anisotropy is that some of these components are null for symmetry reasons. Or, viceversa, if the components that should be null are not, some sort of deformation of the lattice with respect to the ideal situation must be taken into account.

The examples refer to the (hyper)polarizabilities of polyacetylene (PA) for which both electronic and ionic contributions are important, the NLO properties of benchmarks as urea $\left(\mathrm{CH}_{4} \mathrm{~N}_{2} \mathrm{O}\right)$ and potassium di-hydrogen phosphate (KDP, $\mathrm{KH}_{2} \mathrm{PO}_{4}$ ), and the birefringence of a series of uniaxal and biaxal minerals. 


\subsection{The case of polyacetylene: the importance of the ionic contribution}

The electronic (hyper)polarizabilities (with zero-point averaging included) which are calculated from the SC-CP method described above do not take into account the socalled pure vibrational effects, which can be quite important. In the CRYSTAL code, these ionic effects are taken into account analytically for the polarizability, $\boldsymbol{\alpha}$ (Eq. 12), and for the first hyperpolarizability, $\boldsymbol{\beta}$ (Eq. 6 of Rérat et al. 2015), from the IR and Raman spectra, but not for $\gamma$, the second hyperpolarizability (hyper-Raman data are also required; see Champagne et al. 1997).

Also the dynamic $\boldsymbol{\alpha}^{e}(\omega)$ (or $\boldsymbol{\alpha}^{e}(-\omega ; \omega)$ ) and $\boldsymbol{\beta}^{e}\left(-\omega_{\sigma} ; \omega_{1}, \omega_{2}\right)$ (but not the corresponding $\left.\boldsymbol{\gamma}^{e}\right)$ can be obtained from CRYSTAL.

The finite field (FF) scheme was implemented in the CRYSTAL code before SC-CP, to compute numerically the static response properties of molecules and periodic systems (see Darrigan et al. 2003). Using then, the finite field nuclear relaxation (FF-NR) scheme of Bishop et al. (1995) that mixes the FF and SC-CP methods, several NLO processes due to the second hyperpolarizabilities can be studied by fitting the Taylor developments of the dipole moment, polarizability, and first hyperpolarizability with respect to the static finite field $\mathbf{E}$, at the equilibrium geometry optimized in the presence of the field or not, $\mathbf{R}_{E}$ and $\mathbf{R}_{0}$ respectively. For example, the Taylor development of the SC-CP (electronic) polarizability is:

$$
\begin{aligned}
\alpha_{t u}^{e}\left(\mathbf{R}_{0}, \mathbf{E}\right)= & \alpha_{t u}^{e}\left(\mathbf{R}_{0}, \mathbf{0}\right)+\sum_{v} \beta_{t u v}^{e} \mathrm{E}_{v} \\
& +\frac{1}{2} \sum_{v, w} \gamma_{t u v w}^{e} \mathrm{E}_{v} \mathrm{E}_{w}+\cdots
\end{aligned}
$$

$$
+\frac{1}{2} \sum_{v, w} g_{\text {tuvw }}^{\alpha} \mathrm{E}_{v} \mathrm{E}_{w}+\cdots
$$

where:

$b_{t u v}^{\alpha}=\beta_{t u v}^{e}+\left.\beta_{t u v}^{n r}(-\omega ; \omega, 0)\right|_{\omega \rightarrow \infty}$

$g_{\text {tuvw }}^{\alpha}=\gamma_{\text {tuvw }}^{e}+\left.\gamma_{\text {tuvw }}^{n r}(-\omega ; \omega, 0,0)\right|_{\omega \rightarrow \infty}$.

The superscript $n r$ indicates the nuclear relaxation approximation for the (field-free) equilibrium vibrational contribution, and the (circular) frequencies of the applied fields are given (as usual) in parentheses, e.g.: $\boldsymbol{\beta}(0 ; 0,0)=\boldsymbol{\beta}\left(-\omega_{\sigma} ; \omega_{1}, \omega_{2}\right)$ with static applied fields $\omega_{i}=0$ and $\omega_{\sigma}=\omega_{1}+\omega_{2}$.
Note that the fits on the dipole moment with respect to the static field should lead, in principle, to linear terms identical to the electronic and total static polarizabilities, the latter being the sum of the electronic (Eq. 10) and nuclear relaxation (Eq. 12) contributions to the polarizability:

$\boldsymbol{\alpha}^{\mu}=\alpha^{e+n r}=\boldsymbol{\alpha}^{e}+\boldsymbol{\alpha}^{n r}$,

since, in either case, only harmonic vibrational terms are included. However, the static hyperpolarizabilities also contain contributions due to anharmonic force constants and anharmonic electrical property derivatives (see, for example, Torrent-Sucarrat et al. 2004). To isolate the nuclear relaxation term, one can either subtract the analytically determined electronic term or calculate the difference between numerical values from the Taylor developments with and without geometry optimization in the presence of the static field. When the geometry is not specified, it is $\mathbf{R}_{0}$; the omitted frequencies are zero. The subscript $\omega \rightarrow \infty$ in Eqs. 29 and 30 refers to the infinite optical frequency (high-frequency or UV-visible frequency) approximation. In addition to harmonic terms, first-order anharmonic contributions are also included for $\left.\gamma^{n r}(-\omega ; \omega, 0,0)\right|_{\omega \rightarrow \infty}$ with this FF-NR method (see Bishop et al. 1995); for the other two NLO processes, $\left.\boldsymbol{\beta}^{n r}(-\omega ; \omega, 0)\right|_{\omega \rightarrow \infty}$ and $\left.\gamma^{n r}(-2 \omega ; \omega, \omega, 0)\right|_{\omega \rightarrow \infty}$ (the latter being obtained from fits of $\left.\boldsymbol{\beta}^{e}\right)$, the first-order anharmonicity terms vanish.

The measured values of non-linear optical properties ordinarily correspond to the sum of vibrational and electronic contributions. In principle, the two may be separated experimentally as well as computationally. For the former, this requires frequency-dependent measurements, as discussed above for $\boldsymbol{\alpha}$, and implied when passing for hyperpolarizabilities as suggested by Shelton (1986).

Let us consider now the case of all-trans polyacetylene (PA) (Lacivita et al. 2012), lying in the $x y$ plane, with alternating double and single $\mathrm{C}-\mathrm{C}$ bonds along the $x$ periodic direction (a double $\mathrm{C}-\mathrm{C}$ bond is included within each unit cell). The center of the unit cell is an inversion point (which annihilates odd order energy perturbation terms, i.e., $\mu$ and $\beta$ in our case) and lies on a $C_{2}$-axis perpendicular to the $\sigma_{h}^{x y}$ mirror plane, which relates $z$ and $-z$ directions, so that all the components of the $\alpha$ and $\gamma$ tensors containing an odd number of $z$ indices vanish.

A finite field is applied along the non-periodic directions $(y, z$, and mixed $y z)$ to obtain the various non-zero independent tensor components of the vibrational (hyper)polarizabilities. $\gamma$ is a fourth-order tensor consisting, in principle, of $3^{4}=81$ components, $\gamma_{t u v w}$. Several components are, however, null or equivalent either by point symmetry or permutation of indices, the latter depending on the number of static field indices. For example, in the case of $\left.\gamma_{t u V W}^{n r}(-\omega ; \omega, 0,0)\right|_{\omega \rightarrow \infty}$, only the permutations $\mathscr{P}_{t, u}$ (associated with the $\omega \rightarrow \infty$ 
limit) and $\mathscr{P}_{V, W}$ (between two static fields) leave the property invariant. According to the number ( $m=4,2$ or 1 ) of static fields, there are three nuclear relaxation contributions to the second hyperpolarizability:
(a) $\gamma_{4, t u v w}^{n r}=\gamma_{T U V W}^{n r}(0 ; 0,0,0)$,
(b) $\gamma_{2, t u v w}^{n r}=\left.\gamma_{t u v W}^{n r}(-\omega ; \omega, 0,0)\right|_{\omega \rightarrow \infty}$ and
(c) $\gamma_{1, t u v w}^{n r}=\left.\gamma_{t u v W}^{n r}(-2 \omega ; \omega, \omega, 0)\right|_{\omega \rightarrow \infty}$.

Seven different finite fields were applied along each direction $(y, z$ and $y=z)$ in Lacivita et al. (2012), namely $|\mathbf{E}|=0.1$, $0.5,1,2.5,5,7.5,10 \times 10^{-3}$ a.u.. Overall, 21 field-dependent geometry optimizations, $\mathbf{R}_{\mathbf{E}}$, followed by the SC-CP1 calculations at the first order of perturbation were used to generate $\boldsymbol{\mu}\left(\mathbf{R}_{\mathbf{E}}\right), \boldsymbol{\alpha}^{e}\left(\mathbf{R}_{\mathbf{E}}\right)$ (left hand side of Eq. 28) and $\boldsymbol{\beta}^{e}\left(\mathbf{R}_{\mathbf{E}}\right)$. To extract the nuclear relaxation (hyper)polarizabilities from the Taylor expansions of the dipole moment and (hyper) polarizabilities (right-hand side of Eqs. 29 and 30 for the polarizability), an additional set of SC-CP2 calculations at the second-order of perturbation was performed at the fieldfree optimized geometry, $\mathbf{R}_{0}$. Total and nuclear relaxation contributions to $\gamma$ are reported in Table 1 .

Let us consider first the transverse (in-plane and non-periodic) yyyy-component of $\gamma$. The vibrational contribution to the static value, $\gamma_{4}^{n r}(0 ; 0,0,0)=1169$ a.u. for four static fields, is almost $50 \%$ of the total value, $g^{\mu}(0 ; 0,0,0)=2419$ a.u., while this percentage decreases to $25 \%$ and $-2 \%$ when only two or one fields are static, $\gamma_{2}^{n r}(-\omega ; \omega, 0,0)$ and $\gamma_{4}^{n r}(-2 \omega ; \omega, \omega, 0)$, respectively. The same comment applies to components including the out-of-plane $z$-direction and off-diagonal $y z$ indices.
In the longitudinal $x$-direction of PA, no components of $\gamma$ can be calculated from the fit of the dipole moment with respect to static fields, since its $\mu_{x}$-component is ill-defined. Then, only components of $\gamma$ having 1,2, or $3 x$-indices can be obtained from fits of $\alpha_{x y(z)}^{e}$ and $\beta_{x y z}^{e}, \alpha_{x x}^{e}$ and $\beta_{x x y(z)}^{e}$, and $\beta_{x x x}^{e}$ with respect to static $\mathrm{E}_{y}$ and $\mathrm{E}_{z}$ fields. This means that at least one field must be frequency-dependent, leading then to a small nuclear relaxation contribution $\gamma_{1}^{n r}$ to $g^{\beta}$ with respect to the electronic one. However, the vibrational contribution is larger than the electronic one for the two-static field $g^{\alpha}$ non-linear optic property, particularly when both in-plane periodic $x$ and non-periodic $y$ components are involved. In the case of $\gamma_{x x y y}(-\omega ; \omega, 0,0)$, the vibrational contribution, $\gamma_{2}^{n r}$, is equal to $+2.332 \times 10^{4}$ a.u., while the total value is smaller: $g^{\alpha}=+9877$ a.u., showing that the electronic contribution has an opposite sign with respect to the vibrational contribution, being equal to $-1.334 \times 10^{4}$ a.u..

It is worth noting that $\gamma_{x x x x}^{n r}$ for the intensity-dependent refractive index (IDRI) process depends on the Raman intensities only, as $\alpha_{x x}^{n r}$ depends on the IR intensities, and can be directly obtained as follows (Champagne et al. 1997):

$\gamma_{x x x x}^{n r}(-\omega ; \omega,-\omega, \omega)=2 \sum_{i=1}^{3 N-6} \frac{\left(\frac{\mathrm{d} \alpha_{x x}^{e}}{\mathrm{~d} Q_{i}}\right)^{2}}{\omega_{i}^{2}-\omega^{2}}$,

The CRYSTAL code computes the Raman intensities, and then allows to analytically obtain this parallel IDRI vibrational contribution too, which is of the same order of magnitude as the electronic one, around $6 \times 10^{6}$ a.u. at the HF level of calculation with a 6-31G basis set including ghost atoms (basis set B in Lacivita et al. 2012).
Table 1 FF-NR static and dynamic vibrational (nuclear relaxation) contributions to the second hyperpolarizability $\gamma$ (in a.u.) of PA obtained by fitting (a) the dipole moment, (b) the polarizability and (c) the first hyperpolarizability versus the finite field (according to Eq. 28 in the polarizability case)
Fitted values

\begin{tabular}{|c|c|c|c|c|c|c|}
\hline \multirow[t]{2}{*}{ 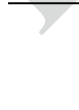 } & \multicolumn{2}{|l|}{ (a) } & \multicolumn{2}{|l|}{ (b) } & \multicolumn{2}{|l|}{ (c) } \\
\hline & $\overline{g^{\mu}}$ & $\gamma_{4}^{n r}$ & $\overline{g^{\alpha}}$ & $\gamma_{2}^{n r}$ & $\overline{g^{\beta}}$ & $\gamma_{1}^{n r}$ \\
\hline yyyy & $2419 \pm 44$ & 1169 & $1672 \pm 26$ & 422 & $1223 \pm 8$ & -27 \\
\hline$z z z z$ & $2843 \pm 283$ & 371 & $2780 \pm 16$ & 308 & $2514 \pm 6$ & 42 \\
\hline$x x x y$ & - & - & - & - & $(1.373 \pm 0.004) \times 10^{5}$ & 2900 \\
\hline xyyy & - & - & $1675 \pm 80$ & 5247 & $-3614 \pm 5$ & -42 \\
\hline xxyy & - & - & $9877 \pm 1009$ & $2.332 \times 10^{4}$ & $(-1.358 \pm 0.002) \times 10^{4}$ & -140 \\
\hline$x x z z$ & - & - & $(1.158 \pm 0.241) \times 10^{4}$ & 5874 & $5766 \pm 7$ & 60 \\
\hline$y y z z$ & $1541 \pm 17$ & 933 & $861.3 \pm 15.7$ & 253.2 & $658.1 \pm 3.1$ & 50 \\
\hline$z z y y$ & $=y y z z$ & $=y y z z$ & $671.7 \pm 7.3$ & 63.7 & $602.6 \pm 0.9$ & -5.5 \\
\hline$y z y z$ & $=y y z z$ & $=y y z z$ & $1855 \pm 31$ & 1247 & $=y y z z$ & $=y y z z$ \\
\hline$x y z z$ & - & - & $879.4 \pm 202.8$ & 659.5 & $250.2 \pm 1.9$ & 30.3 \\
\hline$x z y z$ & - & - & $2740 \pm 86$ & 2520 & $=x y z z$ & $=x y z z$ \\
\hline$x z z y$ & - & - & $=x z y z$ & $=x z y z$ & $220.0 \pm 0.1$ & 0.1 \\
\hline
\end{tabular}

HF Hamiltonian and 6-31G(d) basis set including ghost atoms

\begin{tabular}{|l|l|l|l|l|}
\hline Journal : Large 12210 & Article No : 931 & Pages : 17 & MS Code : 931 & Dispatch : 20-7-2020 \\
\hline
\end{tabular}


The static $\gamma_{x x x x}^{n r}(0 ; 0,0,0)$ term, instead, needs also the determination of the product of IR and hyper-Raman amplitudes, $\left(\mathrm{d} \mu_{x} / \mathrm{d} Q_{i}\right)\left(\mathrm{d} \beta_{x x x}^{e} / \mathrm{d} Q_{i}\right)$, to be evaluated at the equilibrium geometry. The latter is not yet available in the CRYSTAL code. Nevertheless, in the polyacetylene case, the IR intensity is small, leading to $\alpha_{x x}^{n r} \ll \alpha_{x x}^{e}$ [0.2 and 171.5 a.u., respectively (Lacivita et al. 2012)] and then most probably to a small contribution, such that the very large $\gamma_{x x x x}^{n r}(0 ; 0,0,0)$ value can be evaluated from Eq. 32 with $\omega=0$.

\subsection{KDP and urea: dielectric tensor $\epsilon^{e}$, second-harmonic generation $\mathrm{d}^{e}$, and the effect of the field wavelength $\lambda$}

In the previous example of polyacetylene, the subscript $\omega \rightarrow \infty$ in Eqs. 29 and 30 means that only the electronic transitions, the energies of which are much larger than the IR mode ones, are involved in the corresponding high-frequency ("infinite frequency") electric field perturbation, but the frequency was set to zero in the calculation. In the present section, the effect of the field wavelength is studied on the electronic contribution to the so-called high-frequency or optical dielectric tensors.

Let us consider the examples of tetragonal KDP and urea. In Table 2, the non-null components of the optical dielectric tensor and SHG susceptibility (electronic contribution only, $\boldsymbol{\epsilon}^{e}$ and $\left.\mathbf{d}^{e}\right)$ at zero frequency, as obtained at the HF level and with various DFT functionals, are reported, and compared with the experimental determinations at $\lambda=1064 \mathrm{~nm}$ wavelength. The ionic contribution to electric properties at this wavenumber is negligible, but the corresponding photon energy $(\sim 1 \mathrm{eV})$ is by far smaller than the gap value (and the UV-visible absorption edge), confirming that it can be considered as null in the electronic contribution as we will see further: $\boldsymbol{\epsilon}_{\lambda=1064 n m}^{e+n r} \sim \boldsymbol{\epsilon}_{\lambda \rightarrow \infty}^{e}$.

$\epsilon$ is a symmetric second-order tensor; only two components, $\epsilon_{x x}$ and $\epsilon_{z z}$, are independent and non-null for symmetry reasons. As regards the third-rank SHG $\mathbf{d}=\frac{1}{2} \chi^{(2)}$, only one non-equivalent component ( $x y z)$ survives. It should be underlined that part of the reduction of the number of independent terms is due to the intrinsic symmetry of the physical property (for $\epsilon$, its symmetric character reduces the constants from 9 to 6 ; in a similar way, the intrinsic symmetry of SHG reduces, for a triclinic compound, the non-null and non-equivalent terms from 27 to 10). In the last line of Table 2, the band gap is also reported, due to its relevance in determining the numerical values of the various quantities.

Tables 2 and 3 permit to discuss two points characterizing the simulation of the reported properties, and of the SC-CP approach:

(a) the effect of the adopted functional;

(b) the effect of the self-consistent treatment.

As regards point (a), in the tables, the results obtained with five of the most popular approaches, namely HF, LDA (Perdew and Zunger 1981), PBE (Perdew et al. 1996), PBE0 (Adamo and Barone 1999), and B3LYP (Becke 1993; Lee et al. 1988), are reported. The HF Hamiltonian is known to be affected by a universal overestimation of the band gap $E_{g}(15.99 \mathrm{vs} 7.12 \mathrm{eV},+125 \%)$. This leads to a systematic underestimation of the dielectric properties $\left(-10 \%\right.$ for $\epsilon_{x x}^{e}$, $-49 \%$ for $d_{x y z}^{e}$ ): remember that CPHF and CPKS are SC-CP perturbative schemes in which the gap appears in the denominator in the analytical definition of the optical properties, see, for example, Eq. 10 where $\omega_{n} \geq$ gap.

At the other extreme, the LDA energy gap is underestimated (5.72 vs $7.12 \mathrm{eV},-20 \%$ ), because of the self-interaction error; as a consequence, also the (hyper)polarizabilities are generally overestimated ( $+4 \%$ for $\epsilon_{x x}^{e},+25 \%$ for $d_{x y z}^{e}$ ).

Gradient corrections (e.g., PBE) provide only small improvements for the gap $(-16 \%)$, and for the dielectric properties $\left(+4 \%\right.$ for $\epsilon_{x x}^{e},+20 \%$ for $\left.d_{x y z}^{e}\right)$.

When the two hybrid functionals, B3LYP $(+12 \%$ for the gap) or PBE0 $(+20 \%$, about the same error, with opposite sign, of LDA), are used, the difference with respect to experiment is usually smaller than when using LDA or PBE ( $0 \%$ for $\epsilon_{x x}^{e},-4 \%$ for $d_{x y z}^{e}$ for B3LYP, $-1 \%$ for $\epsilon_{x x}^{e}$,
Table 2 Coupled-perturbed optical dielectric constants, $\epsilon_{x x}^{e}$ and $\epsilon_{z}^{e}$, and SHG susceptibility, $d_{x y z}^{e}($ in $\mathrm{pm} / \mathrm{V}$ ), of tetragonal KDP (space group I $\overline{4} \mathrm{~d} 2$ ) at zero frequency and different levels of theory

\begin{tabular}{lllllll}
\hline & HF & PBE0 & B3LYP & PBE & LDA & Exp. \\
\hline$\epsilon_{x x}^{e}$ & 2.025 & 2.223 & 2.230 & 2.328 & 2.340 & $2.23^{\mathrm{a}}$ \\
& $(1.712)$ & $(2.195)$ & $(2.244)$ & $(2.526)$ & $(2.562)$ & \\
$\epsilon_{z z}^{e}$ & 1.868 & 2.039 & 2.046 & 2.132 & 2.159 & $2.13^{\mathrm{a}}$ \\
$d_{x y z}^{e}$ & $(1.639)$ & $(2.043)$ & $(2.086)$ & $(2.317)$ & $(2.369)$ & \\
& 0.197 & 0.355 & 0.373 & 0.467 & 0.488 & $0.38^{\mathrm{b}}$ \\
$E_{g}$ & $(0.066)$ & $(0.302)$ & $(0.341)$ & $(0.609)$ & $(0.647)$ & \\
\hline
\end{tabular}

A split valence basis set was used with $\mathrm{d}$ functions on $\mathrm{H}$ and f-functions on $\mathrm{K}, \mathrm{P}$, and $\mathrm{O}$. SOS (sum over states) values in parentheses. $E_{g}$ is the energy gap (in eV). Calculated data from Lacivita et al. (2009). Experimental values from ${ }^{\text {a }}$ Polyanskiy (2020) and ${ }^{\mathrm{b}}$ Eckardt and Byer (1991) at $\lambda=1064 \mathrm{~nm}$ 
Table 3 High-frequency dielectric tensor components $\epsilon_{x x}^{e}$ and $\epsilon_{z z}^{e}$ of urea (three top lines) and KDP (three bottom lines) computed using various Hamiltonians at $\lambda \rightarrow \infty$ limit and $\lambda$ equal to 1064 and $600 \mathrm{~nm}$

\begin{tabular}{|c|c|c|c|c|c|c|c|c|c|}
\hline & $\lambda(\mathrm{nm})$ & & $\mathrm{HF}$ & PBE0 & B3LYP & LC-BLYP & PBE & LDA & Exp. \\
\hline \multirow[t]{6}{*}{ Urea } & \multirow[t]{2}{*}{$\infty$} & $\epsilon_{x x}^{e}$ & 1.901 & 2.059 & 2.070 & 2.057 & 2.149 & 2.186 & \\
\hline & & $\epsilon_{z z}^{e}$ & 2.187 & 2.433 & 2.451 & 2.430 & 2.567 & 2.599 & \\
\hline & \multirow[t]{2}{*}{1064} & $\epsilon_{x x}^{e}$ & 1.907 & 2.070 & 2.081 & 2.067 & 2.163 & 2.202 & 2.194 \\
\hline & & $\epsilon_{z z}^{e}$ & 2.195 & 2.448 & 2.467 & 2.446 & 2.588 & 2.621 & 2.529 \\
\hline & \multirow[t]{2}{*}{600} & $\epsilon_{x x}^{e}$ & 1.920 & 2.093 & 2.106 & 2.091 & 2.195 & 2.237 & 2.220 \\
\hline & & $\epsilon_{z z}^{e}$ & 2.214 & 2.483 & 2.504 & 2.481 & 2.635 & 2.671 & 2.577 \\
\hline \multirow[t]{6}{*}{ KDP } & \multirow[t]{2}{*}{$\infty$} & $\epsilon_{x x}^{e}$ & 2.025 & 2.223 & 2.230 & 2.219 & 2.327 & 2.341 & \\
\hline & & $\epsilon_{z z}^{e}$ & 1.868 & 2.039 & 2.046 & 2.049 & 2.132 & 2.160 & \\
\hline & \multirow[t]{2}{*}{1064} & $\epsilon_{x x}^{e}$ & 2.030 & 2.231 & 2.239 & 2.227 & 2.339 & 2.352 & 2.231 \\
\hline & & $\epsilon_{z z}^{e}$ & 1.872 & 2.046 & 2.053 & 2.055 & 2.141 & 2.169 & 2.131 \\
\hline & \multirow[t]{2}{*}{600} & $\epsilon_{x x}^{e}$ & 2.041 & 2.250 & 2.258 & 2.245 & 2.363 & 2.377 & 2.277 \\
\hline & & $\epsilon_{z z}^{e}$ & 1.880 & 2.060 & 2.068 & 2.069 & 2.160 & 2.189 & 2.155 \\
\hline
\end{tabular}

Data from Rérat et al. (2015). Experimental values from Rosker et al. (1985) for urea and Polyanskiy (2020) for KDP
$-8 \%$ for $d_{x y z}^{e}$ for PBE0). Note, however, that if the other component of the dielectric tensor is considered, $\epsilon_{z z}^{e}$, the LDA or PBE results are closer to experiment than the ones of hybrids $(+1 \%, 0 \%,-4 \%,-4 \%$ and $-12 \%$, from left to right in the table).

Let us consider now the effect of the SCF process, which permits to the system to respond to the electric field perturbation [point (b) above]. The self-consistent coupled-perturbed, SC-CP, results can be compared with the uncoupled SOS (sum over states; iteration 0 of the SC-CP process) data shown in parentheses in any second row of the table.

A few comments concerning $\epsilon^{e}$ :

- In all cases, the coupled-perturbed scheme improves the SOS results.

- The larger the distance $\Delta$ from the experiment at the SOS level, the larger the SC-CP correction (SC-CP minus SOS): for HF, $\epsilon_{x x}^{e}$ varies by 0.32 (from 1.71 to 2.03 ) and $\Delta$ from -24 to $-10 \%$; for LDA, at the opposite side of the table, $\epsilon_{x x}^{e}$ decreases from 2.56 to 2.34 and $\Delta$ from +14 to $+4 \%$.

It is interesting to notice that for hybrids, and in particular for B3LYP, the difference between SOS and SC-CP is quite small (0.01 and 0.04 for $\epsilon_{x x}^{e}$ and $\epsilon_{z z}^{e}$ for B3LYP, and 0.03 and 0.00 for PBE0 for the same components, with $\Delta$ always smaller than 4\%). In summary, hybrid functionals seem to require a much smaller correction from the coupling than LDA, PBE, and HF.

The above comments apply also to the SHG $d_{x y z}^{e}$ data; the effects are, however, much larger in percentage. Therefore, for HF, $\Delta$ increases from $-83 \%$ to $-49 \%$; for LDA, it decreases from +66 to $+25 \%$; for B3LYP from -13 to $-4 \%$, with a relatively modest change in absolute value from 0.34 to $0.37 \mathrm{pm} / \mathrm{V}$.
The data shown in Table 2 are static, but they refer to electronic calculations or measurements in which a high (UV-visible) field frequency has been used. In Table 3, we explore, for both urea and KDP, the effect of the field wavelength, for the electronic dielectric tensor (Rérat et al. 2015). Computed SHG results for urea and KDP (see Table 4) show that B3LYP reproduces rather well the experimental values measured at 1064 and $600 \mathrm{~nm}$. For urea, the quasi-isotropic electronic contribution is slightly smaller than the experimental value at $\lambda=1064 \mathrm{~nm}$ (Halbout et al. 1979). At this wavelength, the vibrational contribution may not be completely negligible. For that reason, the double harmonic vibrational $d_{x y z(z x y)}^{n r}(-2 \omega ; \omega, \omega)$ components were calculated at $\lambda=1064 \mathrm{~nm}$ (see Eq. 6 in Rérat et al. 2015); their value is $0.041(0.035) \mathrm{pm} \mathrm{V}^{-1}$ for B3LYP, and has the same sign as the electronic contribution $d_{x y z(z x y)}^{e}(-2 \omega ; \omega, \omega)$. The total B3LYP $d_{x y z(z x y)}^{e+n r}$ value is, then, equal to 1.027 and $1.018 \mathrm{pm} \mathrm{V}^{-1}$ which falls essentially at the outer limit of the error bars for the experimental value: $d_{14}=1.2 \pm 0.1 \mathrm{pm} \mathrm{V}^{-1}$ of Halbout et al. (1979) $\left(d_{x y z}^{e+n r} \approx d_{z x y}^{e+n r}\right.$ for wavelength larger than $600 \mathrm{~nm}$ ). Vibrational anharmonicity and/or temperature effects, which would increase the magnitude of this term, could be among the reasons of the small discrepancy. At $600 \mathrm{~nm}$, the calculated vibrational contribution is four times smaller than at $1064 \mathrm{~nm}$ and, thus, can be neglected. The B3LYP value (1.371 and $1.361 \mathrm{pm} \mathrm{V}^{-1}$ ) in this case is well within the experimental window: $1.3 \pm 0.3 \mathrm{pm} \mathrm{V}^{-1}$ of Bäuerle et al. (1977). Finally, for KDP, the B3LYP electronic value of $0.41 \mathrm{pm} \mathrm{V}^{-1}$ is in perfect agreement with the available experimental reference, i.e., $0.41 \mathrm{pm} \mathrm{V}^{-1}$ (Singh 1986). Again, Table 4 clearly shows the well-known tendency of LDA and GGA functionals to grossly overestimate highorder electric susceptibilities as the value of the wavelength approaches the resonance. It is noteworthy that $d_{x y z}^{e}$ increases at each frequency when the percentage of HF exchange

\begin{tabular}{|l|l|l|l|l|}
\hline Journal : Large 12210 & Article No : 931 & Pages : 17 & MS Code : 931 & Dispatch : 20-7-2020 \\
\hline
\end{tabular}


Table 4 Calculated SHG high-frequency electric susceptibilities $d_{x y z}^{e}$ and $d_{z x y}^{e}$ (in $\mathrm{pm} / \mathrm{V}$ ) of urea and KDP computed using various Hamiltonians at $\lambda \rightarrow \infty$ limit and $\lambda$ equal to 1064 and $600 \mathrm{~nm}$

\begin{tabular}{|c|c|c|c|c|c|c|c|c|c|}
\hline & $\lambda(\mathrm{nm})$ & & $\mathrm{HF}$ & PBE0 & B3LYP & LC-BLYP & PBE & LDA & Exp. \\
\hline \multirow[t]{6}{*}{ Urea } & \multirow[t]{2}{*}{$\infty$} & $d_{x y z}^{e}$ & 0.680 & 0.823 & 0.863 & 0.876 & 0.949 & 1.106 & \\
\hline & & $d_{z x y}^{e}$ & 0.680 & 0.823 & 0.863 & 0.876 & 0.949 & 1.106 & \\
\hline & \multirow[t]{2}{*}{1064} & $d_{x y z}^{e}$ & 0.738 & 0.936 & 0.986 & 0.989 & 1.131 & 1.333 & $1.2 \pm 0.1$ \\
\hline & & $d_{z x y}^{e}$ & 0.737 & 0.934 & 0.983 & 0.988 & 1.128 & 1.329 & \\
\hline & \multirow[t]{2}{*}{600} & $d_{x y z}^{e}$ & 0.894 & 1.279 & 1.371 & 1.333 & 1.824 & 2.243 & $1.3 \pm 0.3$ \\
\hline & & $d_{z x y}^{e}$ & 0.889 & 1.263 & 1.361 & 1.323 & 1.793 & 2.191 & \\
\hline \multirow[t]{6}{*}{ KDP } & \multirow[t]{2}{*}{$\infty$} & $d_{x y z}^{e}$ & 0.198 & 0.354 & 0.373 & 0.342 & 0.467 & 0.487 & \\
\hline & & $d_{z x y}^{e}$ & 0.198 & 0.354 & 0.373 & 0.342 & 0.467 & 0.487 & \\
\hline & \multirow[t]{2}{*}{1064} & $d_{x y z}^{e}$ & 0.207 & 0.378 & 0.397 & 0.363 & 0.505 & 0.530 & $0.38,0.41$ \\
\hline & & $d_{z x y}^{e}$ & 0.207 & 0.378 & 0.396 & 0.363 & 0.504 & 0.529 & \\
\hline & \multirow[t]{2}{*}{600} & $d_{x y z}^{e}$ & 0.228 & 0.438 & 0.464 & 0.416 & 0.607 & 0.642 & \\
\hline & & $d_{z x y}^{e}$ & 0.227 & 0.435 & 0.460 & 0.415 & 0.603 & 0.636 & \\
\hline
\end{tabular}

Data as in Rérat et al. (2015). Experimental data from Levine and Allan (1993), Halbout et al. (1979), Bäuerle et al. (1977) for urea and from Refs. Eckardt and Byer (1991), Singh (1986) for KDP

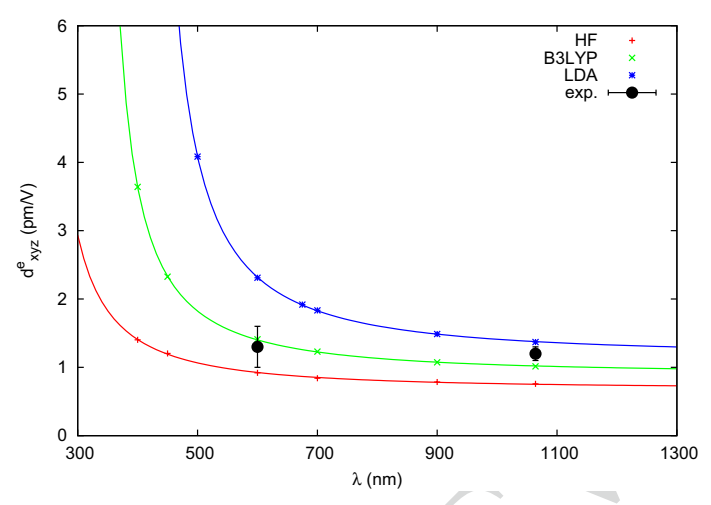

Fig. 2 Variation of the SHG $d_{x y z}^{e}$ tensor component of bulk urea with respect to the field wavelength, $\lambda$, at the HF, B3LYP, and LDA levels of calculation. Experimental data from Levine and Allan (1993), Halbout et al. (1979), Bäuerle et al. (1977) decreases. This correlates with the predicted band gap for the different Hamiltonians. For urea, $E_{g}$ decreases from HF (14.0 $\mathrm{eV})$ to PBE0 (7.4 eV) and B3LYP $(6.9 \mathrm{eV})$ to PBE $(5.2 \mathrm{eV})$ and LDA (4.8 eV); see Table 2 for KDP.

Figure 2 shows the variation of SHG $d_{x y z}^{e}$ as a function of $\lambda$, the field wavelength, for three levels of theory: HF, LDA, and B3LYP. The dots represent the experimental determination. Its error bar is also indicated. The above discussion concerning the $\lambda$ effect becomes here very clear: above 600 (HF), 700 (B3LYP), or 1100 (LDA) $n m d_{x y z}^{e}$ remains essentially constant when the wavelength of the field is varying. As for other properties, B3LYP performs best.

\subsection{Refractive index $n$ and birefringence $\delta$ of minerals}

The refractive index and birefringence are directly related by simple equations to the components of the dielectric tensor, as shown in Sect. 2.2.

Here, we will focus on three aspects:

(a) the relative importance of the electronic and ionic contributions. We remind that the former requires a CPHF calculation at fixed geometry (experimental or calculated); the ionic contribution is more expensive, as it requires to build the full Hessian matrix for generating the vibrational frequencies.

(b) the dependence of these contributions on the wavelength $\lambda$

(c) the anisotropy, that is the different response to the electric field applied in different directions.

As discussed previously, the relative importance of the electronic $\alpha^{e}$ (Eq. 10) and ionic $\alpha^{n r}$ (Eq. 12) contributions to polarizability depends on the frequency. In the UV-visible region, the electronic contribution is much larger than the ionic one. Therefore, we will first consider results obtained at the sodium source wavelength, $\lambda_{D}=589.3 \mathrm{~nm}$, taking into account the electronic contribution alone.

A large set of minerals, namely fluorite $\left(\mathrm{CaF}_{2}\right)$, periclase $(\mathrm{MgO})$, corundum $\left(\mathrm{Al}_{2} \mathrm{O}_{3}\right)$, quartz $\left(\mathrm{SiO}_{2}\right)$, rutile $\left(\mathrm{TiO}_{2}\right)$, anatase $\left(\mathrm{TiO}_{2}\right)$, calcite $\left(\mathrm{CaCO}_{3}\right)$, aragonite $\left(\mathrm{CaCO}_{3}\right)$, andalusite, sillimanite, and kyanite (three $\mathrm{Al}_{2} \mathrm{SiO}_{5}$ polymorphs), forsterite $\left(\mathrm{Mg}_{2} \mathrm{SiO}_{4}\right)$, topaz $\left(\mathrm{Al}_{2} \mathrm{SiO}_{4} \mathrm{~F}_{2}\right)$, and perovskite $\left(\mathrm{CaTiO}_{3}\right)$, plus $\mathrm{CaO}$ have been selected to cover a large range of refractive index and birefringence values. Table 5 reports data calculated for this set of systems, using the B3LYP hybrid functional, relatively severe computational
890

\begin{tabular}{|l|l|l|l|l|}
\hline Journal : Large 12210 & Article No : 931 & Pages : 17 & MS Code : 931 & Dispatch : 20-7-2020 \\
\hline
\end{tabular}


Table 5 Calculated and experimental refractive indices both at $\left(\lambda_{D}\right)$ for various minerals characterized by different symmetry

\begin{tabular}{|c|c|c|c|c|c|c|c|c|c|c|c|}
\hline \multirow[b]{2}{*}{ Ml } & \multirow[b]{2}{*}{$\mathrm{CS}$} & \multicolumn{5}{|c|}{ Experimental data } & \multicolumn{5}{|c|}{ Calculated data } \\
\hline & & $n_{X}$ & $n_{Y}$ & $n_{Z}$ & $\delta$ & $2 V_{Z}$ & $n_{X}$ & $n_{Y}$ & $n_{Z}$ & $\delta$ & $2 V_{Z}$ \\
\hline Flu & $\mathrm{Cub}$ & & 1.4336 & & & & & 1.4325 & & & \\
\hline Per & $\mathrm{Cub}$ & & 1.7355 & & & & & 1.7249 & & & \\
\hline $\mathrm{CaO}$ & $\mathrm{Cub}$ & & 1.8396 & & & & & 1.8205 & & & \\
\hline Cor & Trig & 1.7598 & & 1.7673 & -.0085 & & 1.7326 & & 1.7442 & -.0117 & \\
\hline Qtz & Trig & & 1.5441 & 1.553 & +.0090 & & & 1.5304 & 1.5365 & +.0061 & \\
\hline $\mathrm{Ru}$ & Tet & & 2.613 & 2.900 & +.287 & & & 2.5773 & 2.8447 & +.2674 & \\
\hline Ana & Tet & 2.4889 & & 2.5621 & -.0732 & & 2.4189 & & 2.5147 & -.0958 & \\
\hline $\mathrm{Cal}$ & Rho & 1.486 & & 1.658 & -.172 & & 1.4796 & & 1.6393 & -.1597 & \\
\hline Ara & Ort & 1.530 & 1.681 & 1.685 & -.155 & 162.0 & 1.5245 & 1.6754 & 1.6805 & -.1560 & 160.7 \\
\hline And & Ort & 1.6327 & 1.6387 & 1.6439 & -.0112 & 94.4 & 1.6180 & 1.6254 & 1.6287 & -.0107 & 112.8 \\
\hline Sil & Ort & 1.6576 & 1.6592 & 1.6797 & +.0221 & 31.5 & 1.6378 & 1.6389 & 1.6557 & +.0179 & 28.5 \\
\hline $\mathrm{Ky}$ & Tric & 1.7130 & 1.7221 & 1.7287 & -.0157 & 99.5 & 1.7029 & 1.7114 & 1.7181 & -.0152 & 97.3 \\
\hline Fo & Ort & 1.6358 & 1.6506 & 1.6687 & +.0329 & 85.1 & 1.6112 & 1.6256 & 1.6472 & +.0360 & 79.4 \\
\hline Top & Ort & 1.6107 & 1.6136 & 1.6209 & +.0102 & 66.7 & 1.5920 & 1.5956 & 1.5986 & -.0066 & 95.1 \\
\hline Top & Ort & & & & & & 1.5920 & 1.5944 & 1.5998 & +.0078 & 66.6 \\
\hline $\mathrm{Pv}$ & Ort & nd & nd & nd & $0.018^{*}$ & nd & 2.3440 & 2.3497 & 2.3631 & +.0191 & 66.7 \\
\hline $\mathrm{Pv}$ & Ort & & & & & & 2.3492 & 2.3529 & 2.3790 & $\begin{array}{r}+.0298 \\
\end{array}$ & 42.0 \\
\hline
\end{tabular}

For each mineral (col. 1: Flu: Fluorite, Per: Periclase, CaO, Cor: corundum, Qtz: quartz, Ru: rutile, Ana: anatase, Cal: calcite, Ara: aragonite, And: andalusite, Sil: sillimanite, Ky: kyanite, Fo: forsterite, Top: topaz, Pv: perovskite) the crystalline system is reported (col. 2 : Cub: cubic, Tet: tetragonal, Trig: trigonal, Rho: rhomboedrical, Ort: orthorhombic, Tric: triclinic). Columns 3-5, 6, and 7 report the experimental refractive indices, the birefringence, and the $2 V_{Z}$ angle, respectively. The corresponding calculated values are given in columns 8-10, 11, and 12, respectively. The sign associated with the birefringence $\delta$ is the optical sign. For uniaxial crystals, $n_{Y}=n_{\omega}$ and $n_{Z}=n_{\epsilon}$ for the positive ones and $n_{X}=n_{\epsilon}$ and $n_{Z}=n_{\omega}$ for the negative ones. $2 V_{Z}$ is smaller (larger) than 90 degrees for positive (negative) biaxial compounds. Opening braces indicate sets of polymorphs. Experimental refractive indices from the compilation at Shannon et al. (2002); when several data are available, preference has been given to data agreeing with Fleischer et al. (1984). The accuracy on the refractive experimental indices is $\pm(0.0001-0.0010)$. The variations between different measurements are usually of the order of 0.001 . Opening parentheses associate calculations performed at the experimental (first line) and optimized (second line) geometry

*See text about birefringence of perovskite

conditions, and the basis sets developed by Peintinger et al. (2013). In all but two cases, the experimental geometry has been used. The refractive indices obtained at the optimized geometry are extremely close to the ones computed at the experimental geometry, the exceptions being topaz and perovskite, for which both data are reported in the table. Figure 3 shows the birefringence $(\delta)$ as a function of the refringence expressed by the proxy $\tilde{n}$. In general, being the birefringence a small fraction of the refractive indices, the intermediate refractive index is a good approximation of the refringence. Therefore, $\widetilde{n}=n_{Y}, \tilde{n}=n_{\omega}$, and $\tilde{n}=n$ for biaxial, uniaxial, and cubic crystals, respectively.

Consider first the cubic systems, on the zero axis. For fluorite and $\mathrm{CaO}$, simulation and experiment coincide, whereas, for periclase, the experimental value is slightly larger (1.735 vs 1.725$)$. This is always the case for all systems: when the two circles do not overlap, the experimental $\tilde{n}$ value is always slightly larger than the computed one, the difference being of the order of $2-3 \%$.

Three sets of polymorphs have been considered: (rutile, anatase), (calcite, aragonite), and (andalusite, sillimanite, kyanite). As already observed for the andradite-grossular solid solution (Lacivita et al. 2013), polymorphs with close density (for example, andalusite, sillimanite) present similar indices. When the density is different, the denser system has the largest $\tilde{n}$ value, as is the case of anatase-rutile and of andalusite-kyanite, according to the Gladstone-Dale or Drude law (Anderson and Schreiber 1965). In summary, the $\tilde{n}$ experimental data are well reproduced, and the residual error is small, and always with the same sign.

The agreement between the experimental and calculated birefringence (the difference between the smallest and largest refractive indices) is better than the one for $\tilde{n}$ by about one order of magnitude. This is due to the fact that inaccuracies due to basis set limitations, use of a specific functional, definition of the equilibrium geometry, and numerical approximations are to a large amount the same for the different components of the dielectric tensor, and then cancel when computing birefringence.

The comparison of calculated and experimental optical sign and angle is more delicate. Figure 3 confirms that for uniaxial crystals, the optical sign is correctly predicted, also for quartz or corundum which are characterized by a small birefringence. For corundum, the discrepancy is the largest in the set; note, however, that the experimental birefringence is the smallest in the set. This indicates that the evaluation of the optical sign of weakly birefringent uniaxial crystals is delicate.

For the biaxial crystals, the optical sign and angle are connected. In general, the agreement between calculated and experimental optical signs is good. A closer inspection reveals, however, the difficulty of obtaining the precise shape of the indicatrix, as the interesting case of topaz, orthorhombic, shows. The "module" of the calculated birefringence compares satisfactorily to the experimental one $(0.0102)$ either at the experimental (0.0066) or optimized (0.0078)

\begin{tabular}{|l|l|l|l|l|}
\hline Journal : Large 12210 & Article No : 931 & Pages : 17 & MS Code : 931 & Dispatch : 20-7-2020 \\
\hline
\end{tabular}




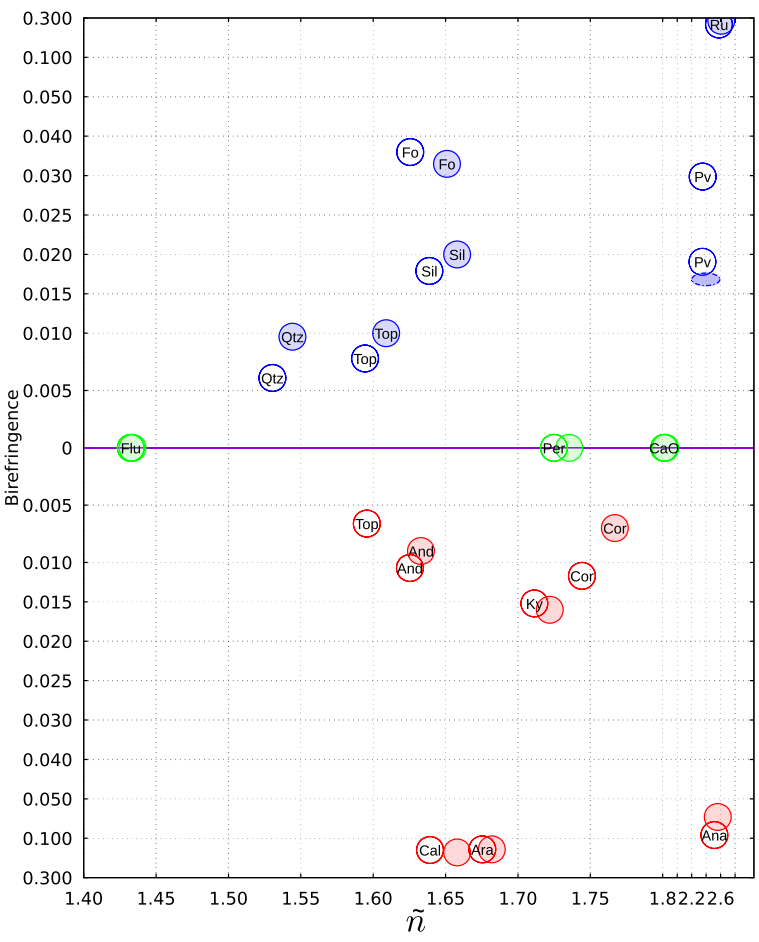

Fig. 3 Birefringence $(\delta)$ versus refringence $(\tilde{n})$. See text for the definition of $\widetilde{n}$, that depends on the crystalline system. Positive and negative uniaxial or biaxial minerals are reported in the upper (blue circles) and lower (red circles) part of the figure, respectively. Open and closed circles correspond to calculated and experimental values, respectively. The ellipse corresponds to perovskite and is centered on the reported interval of indices and the largest measured birefringence (see text); the optical sign is unknown. Abbreviations are as follows: Flu: fluorite, Per: periclase, Cor: corundum, Ru: rutile, Ana: anatase, Cal: calcite, Ara: aragonite, Fo: forsterite, And: andalusite, Sil: sillimanite, Ky: kyanite, Qtz: quartz, Top: topaz, Pv: perovskite. When circles overlap, in all cases, they refer to the same compound, that is indicated only in one of the circles

geometry. However, the sign of the calculated indicatrix reverses according to the considered geometry.

We mention here also the $\mathrm{CaTiO}_{3}$ perovskite case, whose crystals are finely twinned. We are not aware of any experimental determination of the refractive indices, as Table 5 shows. We found, however, a single experimental determination of the birefringence (El-mallah et al. 1987). We indicate this situation with an ellipse, rather than with a circle. The $\delta$ value computed at the experimental geometry is quite close to the experimental value $(0.019$ vs 0.018$)$, whereas the value at the optimized geometry is slightly larger (0.030).

A few words now concern the optical angle $2 \mathrm{~V}$, shown in Table 5, for which we consider again topaz and perovskite. In both cases, the calculated refractive indices at the two geometries are quite close, but the optical angles are different, and strongly dependent on these small differences. The reason is that the size of the indicatrix depends on the refractive index values, whereas the shape is mainly
Table 6 Comparison between calculated and measured refractive indices $n_{\lambda_{D}}$ and $n_{\lambda_{\max }}$

\begin{tabular}{lllllll}
\hline M1 & $\lambda_{D}$ & & & $\lambda_{\max }$ & & \\
\cline { 2 - 3 } & Calc. & Exp. & & Calc. & Exp. & $\lambda_{\max }$ \\
\hline Flu & 1.4325 & 1.4336 & & 1.3001 & 1.3076 & $9724.0^{\mathrm{a}}$ \\
Cor & $1.7442^{\omega}$ & 1.7673 & & 1.5597 & 1.5864 & $5577.0^{\mathrm{b}}$ \\
& $1.7326^{\epsilon}$ & 1.7598 & & $1.5541^{\epsilon}$ & & \\
Per & 1.7249 & 1.7355 & & 1.6100 & 1.6240 & $5350.0^{\mathrm{c}}$ \\
Cal & $1.6392^{\omega}$ & 1.658 & & 1.6057 & 1.6210 & $2170.0^{\mathrm{d}}$ \\
& $1.4796^{\epsilon}$ & 1.486 & & 1.4656 & 1.4739 & $2324.0^{\mathrm{d}}$ \\
Qtz & $1.5304^{\omega}$ & 1.5441 & & 1.5027 & 1.5201 & $2053.1^{\mathrm{d}}$ \\
& $1.5365^{\epsilon}$ & 1.553 & & 1.5103 & 1.5282 & $2053.1^{\mathrm{d}}$ \\
Ru & $2.5773^{\omega}$ & 2.613 & 2.4112 & 2.451 & $1529.6^{\mathrm{e}}$ \\
& $2.8447^{\epsilon}$ & 2.900 & 2.6501 & 2.709 & $1529.6^{\mathrm{e}}$ \\
\hline
\end{tabular}

$\lambda_{\max }$ in $\mathrm{nm}$ in the last column. For uniaxial minerals, superscripts $\omega$ and $\epsilon$ underline that the lines report $n_{\omega}$ and $n_{\epsilon}$, respectively

${ }^{\mathrm{a}}$ Malitson (1963)

${ }^{\mathrm{b}}$ Malitson (1962)

${ }^{\mathrm{c}}$ Stephens and Malitson (1952)

${ }^{\mathrm{d}}$ Ghosh (1999)

${ }^{\mathrm{e}}$ Devore (1951)

controlled by the two partial birefringences: $\delta_{1}=n_{Y}-n_{X}$ and $\delta_{2}=n_{Z}-n_{Y}$. Note that $\delta=\delta_{1}+\delta_{2}$ (Wright 1951). For $\delta<0.05$ and $1.400<n_{X}<2.000$, the main contribution to $\cos 2 V_{Z}$ is given by $\frac{\delta_{2}-\delta_{1}}{\delta}$. The partial birefringence being smaller than the birefringence, small changes of the partial birefringence can change the optical sign. Therefore, at this stage, the calculated sign of the indicatrix of biaxial materials has to be considered as poorly constrained.

As anticipated, at wavelengths shorter than the IR region, the polarizability, and, consequently, the dielectric matrix (Eq. 26) and refractive index (Eq. 16), can be approximated by its electronic part only, since $\alpha^{n r} \sim-\sum_{i} \sigma_{i}^{2} / \omega^{2} \sim 0^{-}$(see Eq. 12 with $\left.\omega \gg \omega_{i}\right)$. However, defining a precise limit for IR is not simple. It depends on the frequency modes $\left(\omega_{i}\right)$ and corresponding Born charges $\left(\sigma_{i}\right)$ of the considered systems. Practically, $4000 \mathrm{~nm}$ (equivalent to $2500 \mathrm{~cm}^{-1}$ ) is a provisional limit that should be applied to most of the studied systems. Hence, experimental indices measured at wavelength shorter than about $4000 \mathrm{~nm}$ should be larger than refractive indices evaluated at infinity, when considering only the electronic part $\left(n_{\lambda \rightarrow \infty}^{e}\right)$, since $\alpha^{e}=\sum_{n} f_{n} /\left(\omega_{n}^{2}-\omega^{2}\right)$ (see Eq. 10) increases with respect to $\omega$ till the first resonance $\omega=\omega_{n}$ in the UV-visible spectrum $(\lambda<600 \mathrm{~nm})$ for many minerals.

Then, we have performed a literature search to find experimental dispersion of the refractive index with respect to the photon energy between the IR and UV-visible absorption spectra $(\lambda \in[200-10,000] \mathrm{nm})$. Available data are represented in Fig. 4. Table 6 reports the largest wavelength $\left(\lambda_{\max }\right)$ at which the refractive index of the

\begin{tabular}{|l|l|l|l|l|}
\hline Journal : Large 12210 & Article No : 931 & Pages : 17 & MS Code : 931 & Dispatch : 20-7-2020 \\
\hline
\end{tabular}




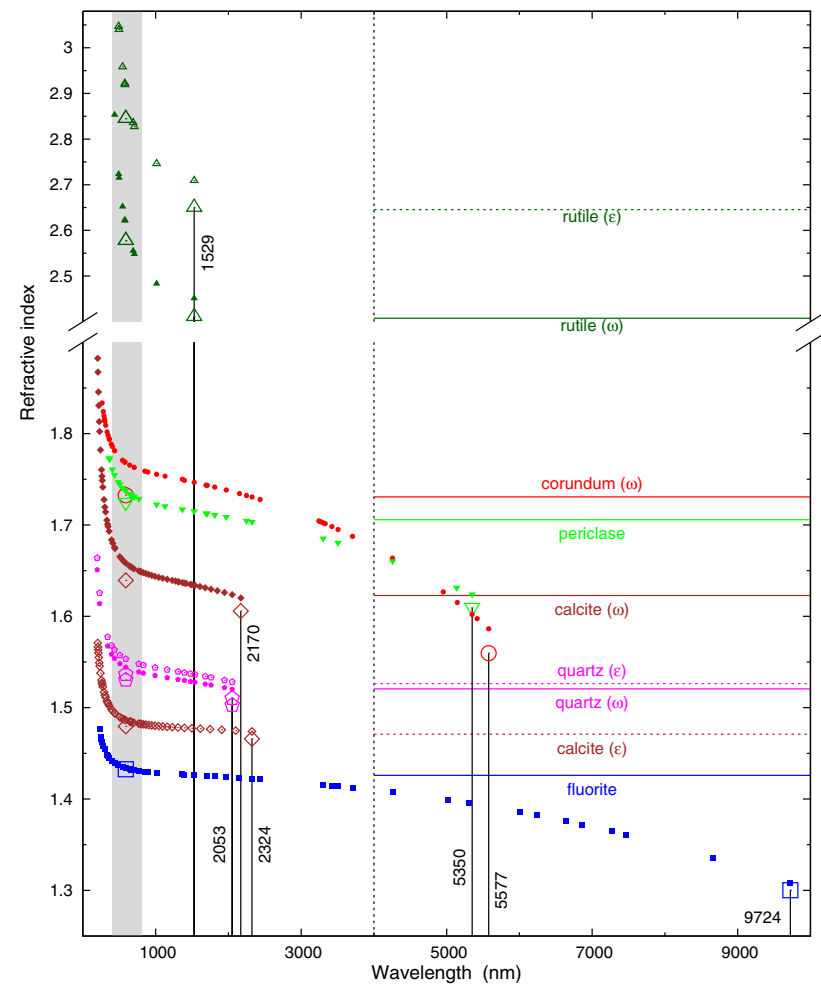

Fig. 4 Refractive index as function of wavelength. Large and small symbols correspond to calculated and measured indices, respectively. Square, circle, downward triangle, upward triangle, diamond, and pentagon correspond to fluorite, corundum, periclase, rutile, calcite, and quartz, respectively. Empty large symbols within and outside the vertical grey strip indicate $n_{\lambda_{D}}$ and $n_{\lambda}^{e+n r}$, respectively. The horizontal lines at height $n_{\lambda \rightarrow \infty}^{e}$ allow to appreciate the role of the ionic contribution to refractive index. For uniaxial crystals, solid and dashed lines refer to $n_{\omega}$ and $n_{\epsilon}$, respectively. $\lambda_{\max }$ is indicated along the vertical line (see Table 6). Color refers to the compound. The grey strip indicates the visible region. The vertical scale of the upper part is half that of the lower part. Notice that no dispersion data have been found for $n_{\epsilon}$ of corundum considered materials have been measured, and the corresponding measured indices $\left(n_{\lambda_{\max }}\right)$, as well as $n_{\lambda_{D}}$, corresponding to the yellow line of $\mathrm{Na}$. For quartz, calcite and rutile, $\lambda_{\max }$ is in the near IR and significantly shorter than $4000 \mathrm{~nm}$. For the first two systems, $n_{\lambda_{\max }}$ is close to the $n_{\lambda \rightarrow \infty}^{e}$ limit value represented by horizontal lines in Fig. 4. For rutile, whose $\lambda_{\max }$ is close to the visible spectrum represented by the vertical grey strip, $n_{\lambda_{\max }}$ is smaller than $n_{\lambda \rightarrow \infty}^{e}$. The difference $n_{\lambda \rightarrow \infty}^{e}-n_{\lambda_{\max }}$ is comparable to its equivalent at $\lambda_{D}$. For fluorite, corundum, and periclase, $\lambda_{\text {max }}$ largely exceeds $4000 \mathrm{~nm}$ and the $n_{\lambda_{\max }}$ values are much smaller than $n_{\lambda \rightarrow \infty}^{e}$, due to the negative ionic contribution, see Eq. 12 when $\omega>\omega_{i}$. The larger $n_{\lambda_{\max }}$, the larger $n_{\lambda \rightarrow \infty}^{e}-n_{\lambda_{\max }}$ (Fig. 4). The difference reaches almost $10 \%$ in the cases of corundum and fluorite and the electronic polarizability alone fails to reproduce indices measured at large wavelengths.
Frequency calculations were performed to evaluate $\alpha^{n r}(\omega)$ (Eq. 12) and the polarizability was evaluated considering the two contributions. In the case of fluorite, corundum and periclase, including the ionic contribution, yield calculated refractive indices $\left(n_{\lambda_{\max }}^{e+n r}\right)$ in very good agreement with the experimental values (Table 6). The difference between calculated and measured indices is now of the order of the difference at $\lambda_{D}$. For rutile, calcite, and quartz, addition of the ionic contribution slightly reduces the calculated indices as expected.

The relative $\alpha^{e}$ and $\alpha^{e+n r}$ contributions to the refractive index in the various spectral regions can be appreciated from Fig. 4 where experimental dispersion data and calculated $n_{\lambda_{D}}, n_{\lambda \rightarrow \infty}^{e}$ and $n_{\lambda_{\max }}^{e+n r}$ are reported for the materials considered. As anticipated above, the electronic contribution yields a fairly good value of the index $n_{\lambda_{D}}$ (large open symbols in the grey vertical strip). However, $n_{\lambda \rightarrow \infty}^{e}$ (horizontal lines) deviate significantly from indices measured at $\lambda>4000 \mathrm{~nm}$. In fact, this "limit" depends on materials. From our data, a better provisional "limit" should be $2500 \mathrm{~nm}$. Above 4000 (or 2500) $\mathrm{nm}$, the ionic contribution cannot be neglected and brings the calculated indices $\left(n_{\lambda_{\max }^{e+n r}}^{e}\right)$ in very good agreement with experimental indices.

\section{Conclusions}

In this document, it has been shown that ab initio quantum mechanical simulation can be used for the calculation of a large set of properties related to the perturbation of an electric field on a crystalline system. The simplest ones, like the dielectric tensor, the refractive indices, the birefringence, correspond to the second order terms in the Taylor expansion of the total energy of the system as a function of the field strength. The laser technology permits nowadays to access experimentally also to the third- and fourthorder terms in the expansion. The combination in various ways of the frequencies of the involved fields multiplies the number of phenomena and physical features that can be accessed experimentally. A large set of these (first and second hyperpolarizability, second-harmonic generation, intensity-dependent refractive indices, and many others) can be obtained from the CRYSTAL code used here, at relatively low cost for small-medium-size periodic systems (the most interesting, due to the tensor nature of many of these quantities).

One of the big advantages of simulation is that the full set of constants defining the tensor are obtained with a single calculation, whereas many experiments must be performed for obtaining the same result.

The combination with other perturbations, for example the strain of the unit cell, generates a new set of important
1063

\begin{tabular}{|l|l|l|l|l|}
\hline Journal : Large 12210 & Article No : 931 & Pages : 17 & MS Code : 931 & Dispatch : 20-7-2020 \\
\hline
\end{tabular}


properties, like the piezoelectric (third order) or photoelastic (fourth order) tensor, available as well in a very simple way from CRYSTAL.

Quantum mechanical simulation appears then an essential tool for the accurate exploration of many tensorial properties of crystalline compounds.

Acknowledgements Access to the HPC resources of CINES/IDRIS/ TGCC obtained thanks to the Grants 2018-[A0040810471] (Fabien Pascale) and 2018-[A0050810537] (Philippe D'Arco) made by GENCI are warmly acknowledged. High-Performance Computing resources were partially provided by the EXPLOR centre hosted by the University de Lorraine. This project has received funding from the ANR (Agence Nationale de la Recherche)-FOIST (project number 18-CE240030-03). Part of this work was granted access to the HPC resources of [CCRT/CINES/IDRIS] under the allocation 2018-2019 and 2019-2020 [A0040807031] made by GENCI (Grand Equipement National de Calcul Intensif). We also acknowledge the Direction du Numérique de l'Université de Pau et des Pays de l'Adour for the computing facilities provided.

\section{References}

Adamo C, Barone V (1999) Toward reliable density functional methods without adjustable parameters: the PBE0 model. J Chem Phys 110(13):6158-6170. https://doi.org/10.1063/1.478522

Anderson OL, Schreiber E (1965) The relation between refractive index and density of minerals related to the Earth's mantle. J Geophys Res 70:1463-1471. https://doi.org/10.1029/JZ070i006p01463

Bäuerle D, Betzler K, Hesse H, Kapphan S, Loose P (1977) Phasematched second harmonic generation in urea. Phys Status Solidi A 42(2):K119-K121. https://doi.org/10.1002/pssa.2210420254

Becke AD (1993) Density-functional thermochemistry. III. The role of exact exchange. J Chem Phys 98(7):5648-5652. https://doi. org/10.1063/1.464913

Bishop DM (1999) Polarizability and hyperpolarizability of atoms and ions. In: Theoretical and computational chemistry. Elsevier, pp 129-146. https://doi.org/10.1016/s1380-7323(99)80007-7

Bishop DM, Hasan M, Kirtman B (1995) A simple method for determining approximate static and dynamic vibrational hyperpolarizabilities. J Chem Phys 103(10):4157-4159. https://doi. org/10.1063/1.469600

Blount EI, Ehrenreich H, Seitz F, Turnbull D (1962) Solid state physics, vol 13. Academic, New York

Boulon G (2001) Nonlinear optics: molecular engineering. ScienceDirect. https://doi.org/10.1016/B0-08-043152-6/01111-6

Boyd RW (2003) Nonlinear optics. Chapter 4: the intensity-dependent refractive index. Elsevier. https://doi.org/10.1016/B978-01212 1682-5/50005-5

Champagne B, Perpète EA, André JM, Kirtman B (1997) Analysis of the vibrational static and dynamic second hyperpolarizabilities of polyacetylene chains. Synth Met 85:1047-1050. https://doi. org/10.1016/S0379-6779(97)80146-4

Condon EU (1937) Theories of optical rotatory power. Rev Mod Phys 9(4):432-457. https://doi.org/10.1103/revmodphys.9.432

Coulson CA (1941) II.- - the Van der Waals force between a proton and a hydrogen atom. Proc R Soc Edinb Sect A Math Phys Sci 61(1):20-25. https://doi.org/10.1017/s0080454100006038

Darrigan C, Rérat M, Mallia G, Dovesi R (2003) Implementation of the finite field perturbation method in the crystal program for calculating the dielectric constant of periodic systems. J Comput Chem 24(11):1305-1312. https://doi.org/10.1002/jcc.10274
Devore JR (1951) Refractive indices of rutile and sphalerite. J Opt Soc Am 41(6):416-419. https://doi.org/10.1364/JOSA.41.000416

Dovesi R, Saunders VR, Roetti C, Orlando R, Zicovich-Wilson CM, Pascale F, Civalleri B, Doll K, Harrison NM, Bush IJ, D'Arco P, Llunell M, Causà M, Noël Y, Maschio L, Erba A, Rérat M, Casassa S (2017) Crystal17 User's Manual. University of Torino. http://www.crystal.unito.it

Dovesi R, Erba A, Orlando R, Zicovich-Wilson CM, Civalleri B, Maschio L, Rérat M, Casassa S, Baima J, Salustro S, Kirtman B (2018) Quantum-mechanical condensed matter simulations with CRYSTAL. Wiley Interdiscip Rev Comput Mol Sci 8(4):e1360. https://doi.org/10.1002/wcms. 1360

Dovesi et al (2020) The CRYSTAL code, 1976-2020 and beyond, a long story.J Chem Phys 152:204111. https://doi.org/10.1063/5.0004892

Eckardt RC, Byer RL (1991) Measurement of nonlinear optical coefficients by phase-matched harmonic generation. In: Bordui PF (ed) Inorganic crystals for optics, electro-optics, and frequency conversion, SPIE, Proc. SPIE, vol 1561. https://doi. org/10.1117/12.50759

El-mallah H, Watts BE, Wanklyn B (1987) Birefringence of CaTiO 3 and $\mathrm{CdTiO}_{3}$ single crystals as fonction of temperature. Phase Transit 9(3):235-245. https://doi.org/10.1080/014115987082423 52

Evarestov RA (2007) Quantum chemistry of solids. The LCAO first principles treatment of crystals. Springer, Berlin

Ferrari AM, Orlando R, Rérat M (2015) Ab initio calculation of the ultraviolet-visible (UV-vis) absorption spectrum, electron-loss function, and reflectivity of solids. J Chem Theory Comput 11(7):3245-3258. https://doi.org/10.1021/acs.jctc.5b00199

Ferrero M, Rérat M, Kirtman B, Dovesi R (2008a) Calculation of first and second static hyperpolarizabilities of one- to threedimensional periodic compounds. Implementation in the CRYSTAL code. J Chem Phys 129(24):244110. https://doi. org/10.1063/1.3043366

Ferrero M, Rérat M, Orlando R, Dovesi R (2008b) The calculation of static polarizabilities of periodic compounds. The implementation in the CRYSTAL code for 1D, 2D and 3D systems. J Comput Chem 29(9):1450-1459. https://doi.org/10.1002/jcc.20905

Fleischer M, Wilcox RE, Matzko JJ (1984) Microscopic determination of the nonopaque minerals. U S Geol Surv Bull. https://doi. org/10.3133/b1627_1984

Garza AJ, Scuseria GE (2016) Predicting band gaps with hybrid density functionals. J Phys Chem Lett 7(20):4165-4170. https://doi. org/10.1021/acs.jpclett.6b01807

Ghosh G (1999) Dispersion-equation coefficients for the refractive index and birefringence of calcite and quartz crystals. Opt Commun 163(1-3):95-102. https://doi.org/10.1016/S0030 $-4018(99) 00091-7$

Halbout JM, Blit S, Donaldson W, Tang C (1979) Efficient phasematched second-harmonic generation and sum-frequency mixing in urea. IEEE J Quantum Electron 15(10):1176-1180. https://doi. org/10.1109/jqe.1979.1069900

Hurst GJB, Dupuis M, Clementi E (1988) Ab initio analytic polarizability, first and second hyperpolarizabilities of large conjugated organic molecules: Applications to polyenes $\mathrm{C}_{4} \mathrm{H}_{6}$ to $\mathrm{C}_{22} \mathrm{H}_{24}$. J Chem Phys 89(1):385-395. https://doi.org/10.1063/1.455480

Kirtman B, Luis JM (2010) On the contribution of mixed terms in response function treatment of vibrational nonlinear optical properties. Int J Quantum Chem 111(4):839-847. https://doi. org/10.1002/qua.22880

Lacivita V, Rérat M, Kirtman B, Ferrero M, Orlando R, Dovesi R (2009) Calculation of the dielectric constant $\epsilon$ and first nonlinear susceptibility $\chi(2)$ of crystalline potassium dihydrogen phosphate by the coupled perturbed Hartree-Fock and coupled perturbed Kohn-Sham schemes as implemented in the CRYSTAL code. J Chem Phys 131(20):204509. https://doi.org/10.1063/1.3267048 
Lacivita V, Rérat M, Kirtman B, Orlando R, Ferrabone M, Dovesi R (2012) Static and dynamic coupled perturbed Hartree-Fock vibrational (hyper)polarizabilities of polyacetylene calculated by the finite field nuclear relaxation method. J Chem Phys 137:014103. https://doi.org/10.1063/1.4731266

Lacivita V, D'Arco P, Orlando R, Dovesi R, Meyer A (2013) Anomalous birefringence in andradite-grossular solid solutions: a quantum-mechanical approach. Phys Chem Miner 40(10):781-788. https://doi.org/10.1007/s00269-013-0612-6

Lee C, Yang W, Parr RG (1988) Development of the Colle-Salvetti correlation-energy formula into a functional of the electron density. Phys Rev B 37(2):785-789. https://doi.org/10.1103/physr evb. 37.785

Levine ZH, Allan DC (1993) Large local-field effects in the second-harmonic susceptibility of crystalline urea. Phys Rev B 48(11):7783-7789. https://doi.org/10.1103/physrevb.48.7783

Malitson IH (1962) Refraction and dispersion of syntheric saphire. J Opt Soc Am 52(12):1377. https://doi.org/10.1364/JOSA.52.00137 7

Malitson IH (1963) A redetermination of some optical properties of calcium fluoride. Appl Opt 2(11):1103-1107. https://doi. org/10.1364/AO.2.001103

Marcaud G, Serna S, Karamanis P, Alonso-Ramos C, Roux XL, Berciano M, Maroutian T, Agnus G, Aubert P, Jollivet A, Ruiz-Caridad A, Largeau L, Isac N, Cassan E, Matzen S, Dubreuil N, Rérat M, Lecoeur P, Vivien L (2020) Third order nonlinear optical susceptibility of crystalline oxide yttria-stabilized zirconia. Photon Res 8(2):110. https://doi.org/10.1364/prj.8.000110

Maschio L, Rérat M, Kirtman B, Dovesi R (2015) Calculation of the dynamic first electronic hyperpolarizability $\beta\left(-\omega_{i} \omega_{1}, \omega_{2}\right)$ of periodic systems. Theory, validation, and application to multi-layer $\mathrm{MoS}_{2}$. J Chem Phys 143(24):244102. https://doi. org/10.1063/1.4937770

McDowell K (1976) Exact static dipole polarizabilities for the excited s states of the hydrogen atom. J Chem Phys 65(7):2518-2521. https ://doi.org/10.1063/1.433455

Nye JF (1985) Physical properties of crystals. Oxford University Press, Oxford

Orlando R, Lacivita V, Bast R, Ruud K (2010) Calculation of the first static hyperpolarizability tensor of three-dimensional periodic compounds with a local basis set: A comparison of LDA, PBE, PBE0, B3LYP, and HF results. J Chem Phys 132(24):244106. https://doi.org/10.1063/1.3447387

Orr B, Ward J (1971) Perturbation theory of the non-linear optical polarization of an isolated system. Mol Phys 20(3):513-526. https ://doi.org/10.1080/00268977100100481

Otto P (1992) Calculation of the polarizability and hyperpolarizabilities of periodic quasi-one-dimensional systems. Phys Rev B 45(19):10876-10885. https://doi.org/10.1103/physrevb.45.10876

Peintinger MF, Oliveira DV, Bredow T (2013) Consistent gaussian basis sets of triple-zeta valence with polarization quality for solidstate calculations. J Comput Chem 34(6):451-459. https://doi. org $/ 10.1002 /$ jcc. 23153

Perdew JP, Zunger A (1981) Self-interaction correction to densityfunctional approximations for many-electron systems. Phys Rev B 23(10):5048-5079. https://doi.org/10.1103/physrevb.23.5048
Perdew JP, Burke K, Ernzerhof M (1996) Generalized gradient approximation made simple. Phys Rev Lett 77(18):3865-3868. https:// doi.org/10.1103/physrevlett.77.3865

Polyanskiy MN (2020) Refractive index database. https://refractive index.info

Rérat M, Ferrero M, Amzallag E, Baraille I, Dovesi R (2008) Comparison of the polarizability of periodic systems computed by using the length and velocity operators. J Phys Conf Ser 117:012023. https://doi.org/10.1088/1742-6596/117/1/012023

Rérat M, Maschio L, Kirtman B, Civalleri B, Dovesi R (2015) Computation of second harmonic generation for crystalline urea and KDP. An ab Initio approach through the coupled perturbed Hartree-Fock/Kohn-Sham scheme. J Chem Theory Comput 12(1):107-113. https://doi.org/10.1021/acs.jctc.5b00791

Rosker M, Cheng K, Tang C (1985) Practical urea optical parametric oscillator for tunable generation throughout the visible and nearinfrared. IEEE J Quantum Electron 21(10):1600-1606. https:// doi.org/10.1109/jqe.1985.1072557

Saleh BEA, Teich MC (1991) Fundamentals of photonics: electrooptics. Wiley, New York. https://doi.org/10.1002/0471213748

Shannon RD, Shannon RC, Medenbach O, Fischer RX (2002) Refractive index and dispersion of fluorides and oxides. J Phys Chem Ref Data 31(4):931-970. https://doi.org/10.1063/1.1497384

Shelton DP (1986) Hyperpolarizability dispersion measured for $\mathrm{Kr}$ and Xe. J Chem Phys 84(1):404-407. https://doi.org/10.1063/1.45015 2

Singh S (1986) CRC handbook of laser science and technology, supplement 2: optical materials. In: Weber MJ (ed) Laser \& optical science \& technology. CRC Press, Boca Raton, pp 147-250

Stephens RE, Malitson IH (1952) Index of refraction of magnesium oxide. J Res Natl Bur Stand 49(4):249-252. https://doi. org/10.6028/jres.049.025

Torrent-Sucarrat M, Solà M, Duran M, Luis JM, Kirtman B (2004) Basis set and electron correlation effects on initial convergence for vibrational nonlinear optical properties of conjugated organic molecules. J Chem Phys 120(14):6346-6355. https://doi. org/10.1063/1.1667465

Traini M (1996) Electric polarizability of the hydrogen atom: a sum rule approach. Eur J Phys 17(1):30-36. https://doi. org/10.1088/0143-0807/17/1/006

Wright FE (1951) Computational of the optic axial angle from the three principal refractive indices. Am Mineral 36(7-8):543-556

Yakovkin IN, Dowben P (2007) The problem of the band gap in LDA calculations. Surf Rev Lett 14:481-487. https://doi.org/10.1142/ S0218625X07009499

Publisher's Note Springer Nature remains neutral with regard to jurisdictional claims in published maps and institutional affiliations.

\begin{tabular}{|l|l|l|l|l|}
\hline Journal : Large 12210 & Article No : 931 & Pages : 17 & MS Code : 931 & Dispatch : 20-7-2020 \\
\hline
\end{tabular}

